\title{
Analysis and Application of Potential Energy Smoothing and Search Methods for Global Optimization
}

\author{
Rohit V. Pappu, Reece K. Hart, and Jay W. Ponder* \\ Department of Biochemistry and Molecular Biophysics, Washington University School of Medicine, \\ St. Louis, Missouri 63110
}

Received: May 15, 1998

\begin{abstract}
Global energy optimization of a molecular system is difficult due to the well-known "multiple minimum" problem. The rugged potential energy surface (PES) characteristic of multidimensional systems can be transformed reversibly using potential smoothing to generate a new surface that is easier to search for favorable configurations. The diffusion equation method (DEM) is one example of a potential smoothing algorithm. Potential smoothing as implemented in DEM is intuitively appealing and has certain appropriate statistical mechanical properties, but often fails to identify the global minimum even for relatively small problems. In the present paper, extensions to DEM capable of correcting its empirical behavior are systematically investigated. Two types of local search (LS) procedures are applied during the reversing schedule from the smooth deformed PES to the undeformed surface. Changes needed to generate smoothable versions of standard molecular mechanics force fields such as AMBER/OPLS and MM2 are also described. The resulting methods are applied in an attempt to determine the global energy minimum for a variety of systems in different coordinate representations. The problems studied include argon clusters, cycloheptadecane, capped polyalanine, and the docking of $\alpha$-helices. Depending on the specific problem, potential smoothing and search (PSS) is performed in Cartesian, torsional, or rigid body space. For example, PSS finds a very low energy structure for cycloheptadecane with much greater efficiency than a search restricted to the undeformed potential surface. It is shown that potential smoothing is characterized by three salient features. As the level of smoothing is increased, unique minima merge into a common basin, crossings can occur in the relative energies of a pair of minima, and the spatial locations of minima are shifted due to the averaging effects of smoothing. Local search procedures improve the ability of smoothing methods to locate global minima because they facilitate the post facto correction of errors due to energy crossings that may have occurred at higher levels of smoothing. PSS methods should serve as useful tools for global energy optimization on a variety of difficult problems of practical interest.
\end{abstract}

\section{Introduction}

Global optimization is an important issue in the characterization of complex systems such as glasses, clusters, and large biomolecules. Techniques have emerged over the past few years, all of which exhibit varying degrees of success in application to well-established global optimization test problems. The current algorithms can be classified into four overlapping categories: (1) deterministic methods, (2) stochastic methods, (3) heuristic methods, and (4) smoothing methods. Selected methods from each of these four categories have been extensively reviewed. ${ }^{1,2}$

Deterministic methods include space covering techniques such as branch-and-bound search, ${ }^{3}$ systematic search methods, ${ }^{4}$ and generalized descent methods. ${ }^{5}$ These methods are useful for small problems or for larger systems with well-established constraints, but will in general fail for large problems due to the exponential increase in the size of the space to be searched.

Stochastic methods include Bayesian statistical models ${ }^{6}$ and simulated annealing. ${ }^{7}$ An underlying theme in many stochastic search procedures is the use of Monte Carlo sampling enhanced by the Metropolis criterion. ${ }^{8}$ Some notable Monte Carlo techniques include reweighting methods such as multicanonical sampling, ${ }^{9}$ Monte Carlo with minimization (MCM),${ }^{10}$ a revision of MCM referred to as "basin hopping", ${ }^{11}$ a molecular dynamics- minimization procedure also related to $\mathrm{MCM},{ }^{12}$ mixed Monte Carlo/stochastic dynamics methods, ${ }^{13}$ and the random kick method. ${ }^{14}$

A widely used stochastic method is simulated annealing 7 which is an important tool for global optimization on rugged energy landscapes. In simulated annealing, the system is coupled to a heat bath which is initially at some high temperature. At high temperature the system is characterized by rapid transitions between high- and low-lying minima. The temperature is then slowly lowered according to a prescribed cooling schedule, and the system is allowed to equilibrate at each level using either a Monte Carlo Metropolis criterion ${ }^{8}$ or molecular dynamics in dynamical simulated annealing. ${ }^{15} \mathrm{~A}$ decrease in temperature is associated with an increased likelihood of occupying low energy states and reduced likelihood of jumping out of minima. The approach is analogous to the slow cooling or annealing of a system through the transition region between the liquid and solid phases. The success of simulated annealing is largely determined by the cooling schedule, the size of the largest barrier on the potential energy surface (PES), and the separation between the global minimum and other lowlying conformations. ${ }^{16,17}$ While simulated annealing has been of limited use in the global optimization of proteins and other biopolymer systems, ${ }^{18}$ it is the de facto standard for refinement 
of X-ray and NMR-determined structures of biomolecules. ${ }^{19}$ Adaptive simulated annealing couples a refined choice of parameters to the Metropolis criterion for increased sampling of important regions of conformational space ${ }^{20}$ and has been applied to predict the conformations of Met-enkephalin and a 14-residue polylysine $\alpha$-helix. ${ }^{21}$ A recent variant of simulated annealing is derived from generalized Tsallis statistics. ${ }^{22}$ The classical Boltzmann machinery can be recovered as a special case of this generalized formalism. Generalized simulated annealing ${ }^{23}$ has been revised to satisfy detailed balance and has been shown to reduce to a steepest descent algorithm at low temperatures. ${ }^{24}$ It is still unclear if the use of generalized statistics will yield significant improvements over the traditional Boltzmann formulation for larger biomolecules.

Many heuristic algorithms are based on a reduction of the global problem into smaller subsets for optimization. The assembly of the smaller optimized parts leads to the final answer. Algorithms of this type include the build-up procedure, ${ }^{25,26}$ constrained systematic search algorithms, ${ }^{27}$ scanning methods which replace exhaustive enumeration with Monte Carlo methods, ${ }^{28}$ and genetic algorithms. ${ }^{29}$ These methods lead to the iterative generation of sets of conformations from which the lowest energy conformer is selected. An important shortcoming of these heuristic methods is the overemphasis of shortrange effects over long-range interactions as determinants of low-energy conformations.

An emerging concept in global optimization is potential smoothing. The basic idea is to analytically transform a multidimensional PES by reducing the number of unique minima and the heights of barriers. Such a transformation can project out a catchment region that may be related to the global minimum. The spirit of smoothing methods is contained in previous work which showed that short-range potentials generate large numbers of local minima, and softening these potentials leads to a reduction in the number of local minima. ${ }^{30,31}$ This conceptual framework has been used in the diffusion equation method (DEM) for potential smoothing developed by Scheraga and co-workers. ${ }^{32}$ Important generalizations to DEM include Gaussian density annealing (GDA and AGDA), and other Gaussian phase packet dynamics methods developed by Straub and co-workers. ${ }^{33-35}$ Methods such as the Monte Carlo minimization (MCM) of $\mathrm{Li}$ and Scheraga ${ }^{10}$ and "basin hopping" of Doye and Wales ${ }^{11}$ fall into the category of PES smoothing, though the mechanism of smoothing is different from the DEM ${ }^{32}$ and AGDA $^{34}$ class of methods discussed in this work.

Smoothing of a PES can also be extended to transform the Gibbs distribution function and is the basis of the packet annealing methods of Shalloway. ${ }^{36}$ The success of a smoothing algorithm for global optimization is contingent upon a connection between the deformed and the undeformed surfaces and sufficient structure underlying the original rough PES. Straub ${ }^{17}$ and Church et al. ${ }^{37}$ have reviewed methods for potential smoothing and compared the efficiency of smoothing algorithms with different types of simulated annealing protocols.

We are initiating a series of systematic studies to establish concepts and quantify metrics to compare and contrast the efficiency, extent of sampling, CPU intensity, and accuracy of specific candidates from the four major classes of global optimization outlined above. In this work we detail methods to modulate diffusional smoothing as a tool for global optimization and use recently developed strategies to generalize its scope for global optimization. ${ }^{38}$ These generalizations come under the category of potential smoothing and search methods which will be referred to as PSS. The basic ideas presented in this work can be adapted to generalized smoothing algorithms such as GDA $^{33}$ and AGDA. ${ }^{34}$ Here we concentrate on tuning the simplest version of potential smoothing, i.e., DEM. The focus of this work is to develop an understanding of the smoothing paradigm by applying it to a cross section of molecular conformation problems. Examples are used to quantitatively illustrate the features of smoothing a rough PES, the limitations of the original smoothing protocol, and the efficiency of search enhanced generalizations on large conformational problems. Applications studied include (i) varying sizes of argon atom clusters, (ii) cycloheptadecane, and (iii) regular conformations of polypeptides.

In the next section we describe in detail the different methods for implementing potential smoothing. This is followed by a description of results from the application of PSS. We conclude with a discussion of the features of potential smoothing, limitations of the methods used in this work, and possible future extensions.

\section{Methods}

All calculations were performed using the TINKER modeling package, which implements a self-contained force field engine providing access to several molecular mechanics force fields. ${ }^{39}$ We use a modified version of the united-atom AMBER/ OPLS $S^{40,41}$ force field in applications of potential smoothing to conformational energy surfaces of polypeptides and clusters of argon atoms. A similarly modified $M M 2^{42}$ parameter set was used in calculations on conformations of cycloheptadecane.

Potential Function and Parametrization. The AMBER/ OPLS and MM2 force fields were modified to obtain analytical solutions of a diffusion equation corresponding to each energy term. Modified AMBER/OPLS or MM2 force fields can be deformed to reduce the number of minima on the potential energy hypersurface. This is done by controlling the level of deformation denoted by an independent parameter, $t$.

The modified AMBER/OPLS force field, which will be referred to as DOPLS for deformable OPLS, is a function of the atomic coordinates and $t$. The DOPLS force field is of the form

$$
V_{\text {total }}=V_{\text {bond }}+V_{\text {angle }}+V_{\text {torsion }}+V_{\text {improper }}+V_{\text {vdw }}+V_{\text {charge }}
$$

The first two terms are bond stretch and valence angle bending energies. Torsional terms characterize barriers for internal bond rotation. A CHARMM-style harmonic improper dihedral term is included in DOPLS to impose planarity at $\mathrm{sp}^{2}$ atoms and chirality of $\mathrm{sp}^{3}$ united atoms. In AMBER/OPLS nonbonded terms include van der Waals energies modeled using a 12-6 Lennard-Jones function and electrostatic energies modeled using a Coulomb potential for charge-charge interactions. Their modification in DOPLS is discussed below.

Since it is easy to compute solutions to a diffusion equation for Gaussian-like initial conditions, energy functions for van der Waals interactions are approximated as a sum of either two or four Gaussians. The functional form of the Gaussian approximation for van der Waals interactions is shown in eq 2.

$$
V_{\mathrm{vdw}}\left(r_{i j}\right)=\sum_{k=1}^{n_{\text {gauss }}} a_{k(i j)} \exp \left(-b_{k(i j)} r_{i j}{ }^{2}\right)
$$

We use a two-Gaussian approximation in this work for each pairwise van der Waals interaction. The Gaussians are centered about the origin and are of opposite sign. Typical van der Waals 
interactions present large repulsive barriers for values of $r_{i j}$ less than the radius of the excluded volume shell. The $r_{i j}=0$ region of a Lennard-Jones 12-6 function is rendered inaccessible in the Gaussian approximation of eq 2 by modulating the height of the repulsive Gaussian, $a_{1(i j)}$. Parameters for the two Gaussian approximation are chosen to fit a canonical Lennard-Jones function with an atomic radius $(\sigma)$ and a well depth $(\epsilon)$ of one. We use values of $\left(a_{1}, b_{1}\right)=(14487.1 \mathrm{kcal} / \mathrm{mol}, 9.05148 \AA)$ and $\left(a_{2}, b_{2}\right)=(-5.55338 \mathrm{kcal} / \mathrm{mol}, 1.22536 \AA)$ for $\sigma=1 \AA$ and $\epsilon=1.0 \mathrm{kcal} / \mathrm{mol}$. The pairwise Gaussian parameters are scaled according to the $\sigma$ and $\epsilon$ values for each pairwise interaction using the values prescribed by the force field. DOPLS parameters also include a small nonzero van der Waals radius on polar hydrogen atoms to avoid fusion of atoms due to favorable electrostatic interactions. Values of $\sigma=0.5 \AA$ and $\epsilon=0.010 \mathrm{kcal} / \mathrm{mol}$ were used for all polar hydrogen atoms. This choice has very little effect on the final energy of reasonable, low-energy structures.

The final component of DOPLS is the inclusion of a $\mathrm{CHARMM}^{43}$ improper dihedral term of the form

$$
V_{\text {improper }}={ }^{1} /{ }_{2} K_{\Theta}\left(\Theta-\Theta_{0}\right)^{2}
$$

This term imposes planarity at $\mathrm{sp}^{2}$ atoms and correct chirality at $\operatorname{sp}^{3} \alpha$-carbon and other tetrahedral atoms. Values of $K_{\Theta}$ were chosen to best reproduce the low-energy regions of the standard AMBER/OPLS trigonometric improper torsional term. The change in functional form was necessary to maintain the desired planarity and to avoid chirality changes on deformed energy surfaces.

These modifications to AMBER/OPLS result in only very small structural and energetic deviations from the original force field for low energy minima on the undeformed, $t=0$, DOPLS potential surface. However, each modification is required for either efficient evaluation or desired limiting behavior as the amount of deformation of the surface increases with increasing $t$.

MM2 substitutes out-of-plane bending for the improper dihedral term and also includes a bond stretch-angle bend cross term. In addition MM2 replaces the Lennard-Jones function with a Buckingham exp-6 function for van der Waals interactions. In smoothing applications, the exp- 6 potential is replaced by a Gaussian approximation similar to the form shown in eq 2. Parameters for the two-Gaussian approximation are chosen to fit the original MM2 function, $290000 \exp \left(-12.5 r^{*} / r\right)-$ $2.25\left(r^{*} / r\right)^{6}$. We use values of $\left(a_{1}, b_{1}\right)=(3423.562 \mathrm{kcal} / \mathrm{mol}$, $9.692 \AA)$ and $\left(a_{2}, b_{2}\right)=(-6.503 \mathrm{kcal} / \mathrm{mol}, 1.585 \AA)$ for $r^{*}=$ $1 \AA$ and $\epsilon=1.0 \mathrm{kcal} / \mathrm{mol}$. The pairwise Gaussian parameters are scaled according to the $r^{*}$ and $\epsilon$ values for each pairwise interaction using the values prescribed by the force field.

Diffusion Equation Method for Smoothing of Potential Functions. In $\mathrm{DEM}^{32}$ a molecular mechanics potential function is deformed by iterative application of a smoothing operator. A multidimensional potential function with its characteristic roughness is transformed to $\mathrm{Y}\left(\mathbf{r}_{1}, \mathbf{r}_{2}, \ldots \mathbf{r}_{n} ; t\right)$ using an operator of the form $\partial \mathrm{Y} / \partial t=\rfloor\left\{\mathbf{r}_{1}, \mathbf{r}_{2}, \ldots \mathbf{r}_{N}\right\} \mathrm{Y}$, where $t$ is a dimensionless parameter that controls the level of smoothing. The operator on the right side is a multidimensional diffusion operator. Here $\mathrm{Y}\left(\mathbf{r}_{1}, \mathbf{r}_{2}, \ldots \mathbf{r}_{n} ; 0\right)=V\left(\mathbf{r}_{1}, \mathbf{r}_{2}, \ldots \mathbf{r}_{n}\right)$ is the original undeformed potential function. The diffusion operator is applied to each of the pairwise molecular mechanics terms individually. We assume that the sum of individually deformed parts is equivalent to the deformed potential energy function. This assumption is accurate for distance-dependent potential functions. If a potential function is written as a sum of pairwise distance- dependent terms, i.e., $V(x)=\sum_{i} \sum_{j<i} V_{i j}\left(\left|x_{i}-x_{j}\right|\right)$, where $\mid x_{i}-$ $x_{j} \mid$ denotes the distance between atoms $i$ and $j$, it can be shown that computing a smoothed form for $V(x)$ requires only the smoothed form for the pair potential $V_{i j}$. ${ }^{44}$ This principle is strictly valid only for pair potentials that depend on the scalar distance between atoms. It can be extended to a sum of distance-dependent molecular mechanics terms provided the distance ranges are the same for each term.

In applying the diffusion equation to molecular mechanics functions as initial conditions for diffusion, the pairwise distance can either be a Cartesian or angular distance. For example, torsional potentials are smoothed in terms of torsional angles instead of the 1-4 distance. Smoothing torsional potentials in terms of torsional angles is qualitatively similar to smoothing an equivalent potential over the 1-4 distance especially if the 1-2 and 1-3 distances are kept fixed. However, the choice of smoothing the torsional potential in terms of angular coordinates is the only tractable way to smooth torsional potentials in Cartesian space when the 1-2 and 1-3 distances are not fixed. Details regarding the smoothing of torsional potentials are presented in Appendix A.

The diffusion equation in one dimension with no sources or sinks is of the form

$$
\frac{\partial \rho}{\partial t}=D \frac{\partial^{2} \rho}{\partial x^{2}}
$$

Here $\rho$ is similar to either temperature in heat conduction or concentration of particles in a diffusion controlled process and $D$ is a diffusion coefficient in units of $\AA^{2}$. Solutions to a diffusion equation can be obtained analytically provided we know the initial value of $\rho$ and the boundary conditions. The general solution to the semiinfinite one-dimensional diffusion equation shown in eq $4 \mathrm{a}$ is

$$
\rho(x, t)=\frac{1}{[4 \pi D t]^{1 / 2}} \exp \left(\frac{-\left(x-x_{0}\right)^{2}}{4 D t}\right)
$$

for $x=x_{0}$ at $t=0$. If $\rho(x, t)$ denotes a one-dimensional distribution of Brownian particles then the rms displacement of the Brownian particles as a function of time is

$$
x_{\mathrm{rms}}^{2}=x_{0}^{2}+2 D t
$$

implying that the mean squared displacement between Brownian particles increases linearly with time. This is an important feature in the use of a diffusion equation formalism for deforming potential functions.

For instance, during diffusion of a DOPLS Gaussian van der Waals interaction term the location of the potential minimum is pushed out to larger values of $r$ as $V_{\mathrm{vdw}}(r)$ is deformed. For a two-Gaussian approximation to the van der Waals potential the location of the potential energy minimum may be written in terms of the Gaussian parameters as $r_{\min }=\ln \left(-b_{1} a_{1} / b_{2} a_{2}\right)$. For nonzero values of the smoothing parameter $t$ the location of the potential energy minimum is shifted and can be written as $r_{\text {min }}=\ln \left(-b_{t 1} a_{t 1} / b_{t 2} a_{t 2}\right)$, where $a_{t k}=a_{k} /(1+4 t)^{3 / 2}$ and $b_{t k}=$ $b_{k} /(1+4 t)$. The ratio $-b_{1} a_{1} / b_{2} a_{2}$ increases with increasing $t$, shifting the location of $r_{\min }$. A qualitative description of potential function smoothing is that pairwise interactions between localized atoms are altered to be interactions between diffuse atoms. On smooth surfaces, atoms are delocalized and the atomic positions are described by probability distributions. Interactions between the average location of atoms can be 
TABLE 1: Characterization of the Diffusion Spaces and Diffusion Coefficients for the Different Energy Terms of a DOPLS Molecular Mechanics Potential ${ }^{a}$

\begin{tabular}{llll}
\hline $\begin{array}{c}\text { energy } \\
\text { term }\end{array}$ & \multicolumn{1}{c}{$\begin{array}{c}\text { diffusion } \\
\text { space }\end{array}$} & $\begin{array}{c}\text { distance } \\
\text { interval }\end{array}$ & \multicolumn{1}{c}{$\begin{array}{c}\text { effective } \\
\text { diffusion coeff }\end{array}$} \\
\hline$V_{\text {bond }}$ & finite & $\left(r_{12_{0},}, r_{12_{\text {max }}}\right)$ & $D_{\text {bond }}=0.000156 \AA^{2}$ \\
$V_{\text {angle }}$ & finite & $\left(r_{13_{0}}, r_{13_{\text {max }}}\right)$ & $D_{\text {angle }}=0.0014 \mathrm{radian}^{2}$ \\
$V_{\text {torsion }}$ & finite & $\left(r_{14(0)}, r_{14(\pi)}\right)$ & $D_{\text {torsion }}=0.022 \mathrm{radian}^{2}$ \\
$V_{\text {vdw }}$ & semiinfinite & $\left(r_{14(0)}, \infty\right)$ & $D_{\text {vdw }}=1.0 \AA^{2}$ \\
$V_{\text {electrostatic }}$ & semiinfinite & $\left(r_{14(0)}, \infty\right)$ & $D_{\text {charge }}=1.0 \AA^{2}$
\end{tabular}

${ }^{a}$ In all calculations $t$ is set to a dimensionless parameter that controls the level of smoothing.

computed using the location and width of the Gaussian distribution for each atom. Interaction between diffuse as opposed to localized atoms leads to a reduction in the combinatorial problem and hence a reduction in the number of accessible minima.

Coordinate Representations. We study applications of potential smoothing in three types of spaces: Cartesian $\left(\Gamma_{\mathrm{vc}}\right)$, torsional $\left(\Gamma_{\mathrm{vt}}\right)$, and rigid body $\left(\Gamma_{\mathrm{vr}}\right)$ space. In $\Gamma_{\mathrm{vt}}$ bonds and angles are kept fixed at their starting positions and conformational changes come about due to changes in torsional angles. The DOPLS potential energy function in $\Gamma_{\mathrm{vt}}$ is written as

$$
V\left(\varpi_{1}, \varpi_{2}, \ldots, \varpi_{n}\right)=V_{\text {torsion }}+V_{\text {improper }}+V_{\mathrm{vdw}}+V_{\text {charge }}
$$

In $\Gamma_{\mathrm{vr}}$ different conformations are distinguished by six rigid body degrees of freedom for each molecule, corresponding to three translations and three rotations. The potential function is of the general form

$$
V\left(\mathbf{r b c}_{1}, \mathbf{r b c}_{2}, \ldots, \mathbf{r b c}_{m}\right)=\sum_{i=1}^{m} \sum_{j<i} \sum_{k=1}^{n_{i}} \sum_{l=1}^{n_{j}} V_{(i, k),(j, l)}
$$

In this work, rigid bodies always correspond to distinct molecules, so the summand denotes nonbonded interactions between atoms on different molecules. $m$ is the number of molecules and $n_{i}$ and $n_{j}$ are the number of atoms in each of the molecules $i$ and $j$, respectively.

Diffusion Coefficients To Modulate Smoothing of Potential Function Terms. In analogy with a classical diffusion equation, each of the molecular mechanics terms represents a different initial condition for diffusion. Individual pairwise energy functions differ in their distance range, energy scales, and pairs of atoms involved. Scaling the disparate potential energy terms may be accomplished by choosing a set of empirical diffusion coefficients to control the rate of diffusion of the different terms. Diffusion coefficients can be estimated based on a solution to the appropriate finite one-dimensional diffusion equations in distance space with the upper and lower bounds stipulated as shown in Table 1. In all of the applications reported here, the bond stretch, angle bending, improper dihedral, out-of-plane bending, and bond-angle cross terms are not smoothed. Diffusion coefficients for the Lennard-Jones and Coulomb terms are set to one since these are nonbonded interactions and the range of these potentials is large. Effective diffusion coefficients for local geometric interactions are scaled relative to values for the nonbonded terms.

The DOPLS force field contains terms that are naturally represented in either distance or angular space. Bond stretch, van der Waals, and electrostatic terms describe pairwise interactions in distance space. Angle bending, improper dihedral, and torsional terms describe interactions in angular space.
In order to correctly scale the smoothing of these terms a mapping of variations in angular space onto variations in distance space is needed. For example, if distances are measured in angstroms and angles in radians, then in order to correctly scale the smoothing of different terms an estimate of the change in radians in angular space in terms of angstroms in distance space is needed. A second consideration is the different ranges of potential functions within the same diffusion space. In distance space, bond stretch terms involve only nearestneighbor distances, while nonbonded functions involve larger distances.

Our analysis of this problem leads to a choice of very small diffusion coefficients for bond stretch and angle bending terms. The limit of diffusion coefficients tending to zero is equivalent to not smoothing the bond and angle terms. Small values for diffusion coefficients reflect two important considerations: (i) the limited range of covalent restraint terms and (ii) the intrinsic nature of restraint terms to impose severe penalties for all deviations from ideal geometry. This is reasonable since the objective is to explore conformational space without any significant rearrangements of covalent geometry, i.e., making or breaking of covalent bonds. A similar reasoning is used to justify the use of undeformed bond-angle cross terms and improper dihedral terms.

Torsional potentials are typically a sum of trigonometric terms that impose multifold barriers. Unlike bond stretch and angle bending terms, torsional terms cannot be ignored since these barriers distinguish between conformations and are considerably smaller than the barriers imposed by violation of covalent bond and angle restraints. Setting $D_{\text {torsion }}=1$ leads to the problem that at fairly small nonzero values for the deformation parameter $t$, torsional barriers vanish leading to a nonphysical exploration of conformational space. Since barriers vanish for small values of $t$, typical reversal protocols used in potential smoothing ${ }^{45,46}$ will not feel these barriers until very small values of $t$ at which point the method may have already committed to a conformation with high torsional energy. One solution would be to recast the torsional potential in terms of a 1-4 distance. ${ }^{46}$ However, a 1-4 distance restraint in $\Gamma_{\mathrm{vc}}$ can become severely nonphysical because covalent bonds and angles are merely undeformed and not rigidly fixed. A simpler method is to compute the rate of diffusion in torsional space which is then used to estimate an effective torsional diffusion coefficient in distance space.

The method of choosing empirical diffusion coefficients for covalent restraint and geometry terms has the desired effect of controlling their smoothing relative to nonbonded terms. The current formalism is a generalization of this technique and allows for increased sampling of conformational alternatives on highly deformed surfaces since torsional terms do get smoothed, albeit slowly. A discussion of the methods used to estimate empirical diffusion coefficients is provided in Appendix B.

Potential Smoothing Protocol. A typical potential smoothing protocol involves the following steps: ${ }^{45-47}$

1. The conformational energy of a starting structure in $\Gamma_{\mathrm{vc}}$, $\Gamma_{\mathrm{vt}}$, or $\Gamma_{\mathrm{vr}}$ is minimized using a local conjugate gradient or second derivative minimization method on the $t=0$ undeformed surface. In $\Gamma_{\mathrm{vt}}$ and $\Gamma_{\mathrm{vr}}$ we use an optimally conditioned quasiNewton method without line searches ${ }^{48}$ and in $\Gamma_{\mathrm{vc}}$ we use a truncated Newton optimization algorithm ${ }^{49}$ with a preconditioned linear conjugate gradient solution of Newton's equations.

2. The value of the control parameter $t$ is slowly increased according to a prescribed smoothing schedule, and conformational energy is minimized at each step using the methods discussed in step 1 . 
3. Smoothing of the conformational energy function is carried out until $t=t_{\mathrm{d}}$, where $t_{\mathrm{d}}$ is the level of smoothing for which all starting structures from step 1 converge to the same structure with the same energy. In previous work, ${ }^{45,46} t_{\mathrm{d}}$ was chosen to be the level at which only a single minimum remains on the deformed surface. This is a special case of our condition to choose $t_{\mathrm{d}}$.

4. Starting at $t=t_{\mathrm{d}}$ the deformation is reduced followed by conformational energy minimization as discussed in step 1. On highly deformed surfaces only a few minima remain, so the local optimizer finds a minimum in the same basin as the starting structure by following the downhill gradient closely. Values of $t$ are reduced in small intervals $\Delta t$ chosen according to a prescribed schedule until $t=0$. The reversal process is a fully deterministic procedure and the final value for the conformational energy at $t=0$ is the DEM estimate of the global energy minimum of the molecule.

This protocol may be repeated for different starting structures at step 1 to show that the chosen $t_{\mathrm{d}}$ is sufficiently large to ensure convergence to the same minimum at $t=t_{\mathrm{d}}$. Starting at $t=t_{\mathrm{d}}$ implies the reversal protocol has forgotten the initial conformation and will follow an invariant deterministic path down to the undeformed surface for a given reversal schedule.

Smoothing Schedule. If $t_{\mathrm{d}}$ is a chosen large value of the deformation parameter for forward smoothing and $n_{\mathrm{d}}$ is the number of points between $t=0$ and $t=t_{\mathrm{d}}$, the smoothing schedule changes the deformation parameter according to the formula

$$
t_{\mathrm{i}}=t_{\mathrm{d}}\left(\frac{i}{n_{\mathrm{d}}}\right)^{S}
$$

for forward smoothing and

$$
t_{\mathrm{i}}=t_{\mathrm{d}}\left(\frac{2 n_{\mathrm{d}}-i}{n_{\mathrm{d}}}\right)^{S}
$$

for the reversal, where $i=1,2,3, \ldots, n_{\mathrm{d}}$ and $2 \leq s \leq 6$ for different applications. For a given value of $s$ increasing $n_{\mathrm{d}}$ increases the number of points sampled at smaller values of $t$. As will be discussed in the results section the optimal choice of values for $n_{\mathrm{d}}$ and $s$ varies from problem to problem.

Compactness Restraints in Smoothing. Most applications of smoothing algorithms have used basin functions or confining potentials to impose compactness conditions during the smoothing process. There are two classes of problems where basin functions have been used in the past. One example is the problem of finding the minimum energy configuration of clusters of Lennard-Jones atoms. In DEM smoothing applications ${ }^{45}$ a basin function of the form $a \exp \left(-b r^{2}\right)$ has been used to keep atoms from drifting out to infinity as $t$ becomes large. The choice of values for $a$ and $b$ results in the application of extremely shallow pairwise Gaussians that keep the system bounded at large values of $t$. Similarly, Ma and Straub ${ }^{33}$ have used a harmonic pair potential to confine the sampling of conformations to the manifold of compact clusters. Basin functions or confining potentials are necessary for noncovalent clusters since they impose boundedness on the problem and are required to keep the calculations numerically stable.

The use of basin functions in application of smoothing to connected systems such as peptides or hydrocarbons is questionable. At large values of deformation, if bonds and angles are undeformed in $\Gamma_{\mathrm{vc}}$ or kept fixed as in $\Gamma_{\mathrm{vt}}$, flexible molecules sample maximally extended structures. For example, at large $t$ the values of the $\phi$ and $\psi$ torsional angles in peptides are typically near $180^{\circ}$. Unlike an argon cluster problem there is no boundedness condition to be imposed. However, basin functions restrict sampling to a set of compact conformations and may bias the results of a smoothing protocol due to limited sampling of conformations and perturbation of the unconstrained smoothed surface. In our applications of potential function smoothing we use basin function restraints for Lennard-Jones clusters and not for peptides and isolated organic molecules.

PSS Methods. An extension of potential smoothing is to include a local search protocol during the reversal schedule to search for alternate low-lying minima. ${ }^{38}$ Local searches allow for corrections to be made to estimates of the global minimum at different levels of smoothing. We consider two types of local searches. One alternative is to perform a search in the vicinity of the local minimum along a randomly chosen direction or along normal modes out of the local minimum. A second method would be to move the system over transitions states into adjacent low-lying minima. A general local search algorithm adapted for either of these two methods is as follows:

1. At some chosen value of $t=t_{1}$ during the reversal we reduce the level of smoothing by $\Delta t$ and find a minimum energy conformation using a local optimization protocol. The energy at the local minimum is stored as $V_{\text {local }}$ and its coordinates are denoted by a vector $\mathbf{R}_{\text {local }}$.

2. The system is moved out of the local minimum either along a set of search directions or to a nearby transition state. The energy of the system at the new location is $V_{\text {excite }}$ and the system coordinates are denoted by $\mathbf{R}_{\text {excite }}$.

3. From $\mathbf{R}_{\text {excite, }}$, the system is moved to an alternate location $\mathbf{R}_{\text {new }}$ by an energy minimization. If $V_{\text {new }}<V_{\text {local }}$, then this is the new energy and the system is retained at the new location $\mathbf{R}_{\text {new }}$.

4. The system is moved from $\mathbf{R}_{\text {new }}$ to explore the vicinity of the new local minimum by repeating steps 2 and 3 until a new lower minimum cannot be found.

5. If a new lower minimum cannot be found, we return to step 1 , reduce the value of $t$, and continue steps 2,3 , and 4 until $t=0$.

The final $t=0$ estimate for the "global" minimum is lower than or equal to the minimum obtained from the DEM protocol without local search.

"Normal Mode" Local Search (NMLS). The algorithm we use for searches in the vicinity of a local minimum is very similar to the "two-stage" method proposed by Nakamura et $a l .{ }^{38}$ In their work, eigenmodes corresponding to the largest eigenvalues of the Hessian computed at a local minimum are followed in order to ensure an uphill climb out of a local minimum. We use a generalization of their protocol to different coordinate representations. In $\Gamma_{\mathrm{vc}}$ the true vibrational normal modes are the appropriate mass-weighted eigenvectors of the Hessian, though this is not strictly true in $\Gamma_{\mathrm{vt}}$ or $\Gamma_{\mathrm{vr}}$. However, we will refer to search along the eigenvectors of the Hessian matrix as "normal mode" local search (NMLS) regardless of the coordinate representation.

A point $k$ along a search direction $i$ that satisfies the condition $V_{i, k-1}>V_{i, k}$, and $V_{i, k-1}>V_{i, k+1}$, where the $V^{\prime}$ 's are the conformational energy values, is chosen to be a new point $\mathbf{R}_{\text {excite }}$ from which to start a minimization to an alternate minimum. The condition suggests apparent downhill progress indicating a possible turning point into a new energy basin. In practice the minimization can occasionally drop the system back into $\mathbf{R}_{\text {local }}$ where it originated.

In $\Gamma_{\mathrm{vc}}$, for connected systems, we use a hybrid scheme for PSS. All minimizations are done in Cartesian space but the 
search directions for a guided climb out of the local minimum are torsional space eigenvectors. The main objective of a local search is to explore conformational space in the vicinity of the local minimum. It is sufficient to explore structures on the manifold of bonds and angles corresponding to $\mathbf{R}_{\text {local }}$, i.e., conformational rearrangements come about from varying torsional angles alone. In $\Gamma_{\mathrm{vr}}$ the Hessian is computed in terms of the $6 n$ rigid body coordinates where $n$ is the number of rigid bodies and the eigenvectors of the $\Gamma_{\mathrm{vr}}$ Hessian are the search directions for NMLS.

Transition State Based Search (TSBS). Consider a system in a local minimum with coordinates $\mathbf{R}_{\text {local }}$ at some level of smoothing $t$. The system can be activated from $\mathbf{R}_{\text {local }}$ to a nearby transition state region and quenched to an alternate local minimum $\mathbf{R}_{\text {new. }}$. A subsequent comparison will choose between $\mathbf{R}_{\text {local }}$ and $\mathbf{R}_{\text {new }}$ depending on which of the two conformational energy values is lower; i.e., the system reverts to $\mathbf{R}_{\text {local }}$ if $V_{\text {local }}$ $<V_{\text {new }}$ or remains in the new metastable state $\mathbf{R}_{\text {new }}$ if $V_{\text {new }}<$ $V_{\text {local}}$. In order to implement such a method we need to be able to locate saddle points starting from a local minimum.

Methods that rely on the use of two adjacent minima to locate intervening saddle points by minimization along an orthogonal direction, by propagating a reaction path ${ }^{50}$ or evolving a contour of tangency, ${ }^{51}$ are not useful here since we know only one minimum. Techniques have been developed to locate transition states using the curvature information at a local minimum. ${ }^{52}$ We use the activation and relaxation technique (ART) of Barkema and Mousseau ${ }^{53}$ to locate saddle points from a minimum in disordered systems. This method is briefly outlined below.

Let $\mathbf{R} \mathbf{1}$ be the system coordinates at a local minimum. The system is initially perturbed from this local minimum by generating a small random displacement away from the minimum, i.e., $\mathbf{R}^{*}=\mathbf{R} \mathbf{1}+\delta$. A saddle point is then reached by iteratively following a new force vector of the form

$$
\mathbf{G}=-\nabla V-(1+\alpha)\{-\nabla V \cdot \Delta \mathbf{R}\} \Delta \mathbf{R}
$$

where $\Delta \mathbf{R}=\mathbf{R}^{*}-\mathbf{R} \mathbf{1}$ is a unit vector parallel to the vector from the original minimum to the new location of the system. $\alpha$ is a positive nonzero parameter chosen so that $0<\alpha<1$ and used to ensure that the system does not remain trapped in the local minimum. The $\mathbf{G}$-vector with $\alpha=0$ points in an uphill direction perpendicular to and away from the local minimum. $\mathbf{G}$ is nonzero away from a local minimum or a saddle point, so the simplest strategy is to start at a local minimum and evolve a trajectory along $\mathbf{G}$ from the local minimum until $\mathbf{G}$ becomes very small. A trajectory-based method can be computationally inefficient. One of the problems in using conjugate gradient minimizers to optimize along the uphill direction parallel to $\mathbf{G}$ is there is no object function associated with the force $\mathbf{G}$. Barkema and Mousseau ${ }^{53}$ suggest the use of a LevenbergMarquardt nonlinear least-squares optimization method designed for following $\mathbf{G}$.

In our experience the simple method of iteratively following $\mathbf{G}$ until it becomes small leads to saddle point regions on an undeformed energy surface for small peptides in $\Gamma_{\mathrm{vt}}$. The same method does not work as well in $\Gamma_{\mathrm{vc}}$ for peptides and hydrocarbons since uphill directions often correspond to an unreasonable disruption of covalent geometries. A potential drawback of the G-vector formalism for our purposes is that the saddle point generated is not necessarily a saddle point adjacent to the local minimum of interest. In using this method in a smoothing algorithm we check for true saddle point convergence by using a truncated Newton method ${ }^{49}$ to refine the location of the ART saddle point.

\section{Results}

The original DEM as well as PS-NMLS and PS-TSBS were applied to conformational energy optimization problems of argon atom clusters, capped polyalanine peptides, cycloheptadecane, and rigid polyalanine helices. Results from these applications are discussed in detail below.

1. Clusters of Argon Atoms in $\boldsymbol{\Gamma}_{\mathrm{vc}}$. A DEM smoothing protocol has previously been applied ${ }^{45}$ to find the global energy minimum of varying cluster sizes of argon atoms. For these systems the interatomic interactions are purely van der Waals interactions. Kostrowicki et al. ${ }^{45}$ have used a three-Gaussian fit to model the interaction between argon atoms for a DEM smoothing study. Two Gaussians represent the Lennard-Jones interactions between argon atoms. A third Gaussian is used as a shallow basin function to keep the clusters bounded during smoothing. In analogous work on potential function smoothing based on annealing an approximate classical distribution, GDA, Ma and Straub ${ }^{33}$ have used a four Gaussian fit for the LennardJones term and a harmonic restraint potential to restrict the GDA sampling to the manifold of compact clusters.

Considerable work has been done toward enumerating the various local and global minima for argon atom clusters of different sizes. ${ }^{11,54}$ For cluster sizes larger than $n=13$ the number of minima has been estimated using a relation of the form $g(n) \approx \exp \left(a+b n+c n^{2}\right)$, where $a=-2.5167, b=$ 0.3572 , and $c=0.0286$ are parameters derived from a fit to results from a full enumeration of all the minima for $n<13$. DEM applied to clusters of size $n=5-19,33$, and 55 yields the global minimum for $n=5-7,11,13-15,33$, and $55 .{ }^{45}$ The lowest energy structures of most argon atom clusters are related to Mackay icosahedra that show a fivefold symmetry. However, there are clusters for which the global minimum is derived from either an fcc symmetry as in $n=38$ or Mark's decahedron as in $n=75,76,77,102,103$, and $104 .^{11}$

For $n=8$, Kostrowicki et al. have shown that DEM yields a dodecahedron of triangle faces which is the global minimum on the undeformed surface of the three Gaussian approximation. On a true Lennard-Jones surface the global minimum is a pentagonal bipyramid with two atoms on the outside. It should be noted that the GDA algorithm of Ma and $\mathrm{Straub}^{33}$ and a refined AGDA protocol ${ }^{55}$ also find global minima for different cluster sizes with varying degrees of success. We applied a PS-NMLS to argon atom clusters using a three-Gaussian approximation and parameters $\epsilon=0.2824 \mathrm{kcal} / \mathrm{mol}$ and $\sigma=$ $3.3610 \AA$. The third attractive Gaussian corresponds to a well depth of $-1.7 \mathrm{kcal} / \mathrm{mol}$ and width of $0.00001 \AA$, which restricts sampling to compact clusters. We studied all clusters of size $n$ $=5-39$ and $n=55$. This list includes clusters not reported in the work of Kostrowicki et al., clusters for which the original DEM does not recover the global minimum, ${ }^{45}$ and certain benchmark "hard" problems such as $n=38{ }^{11,56}$

In our application of DEM, we use $t_{\mathrm{d}}=300.0$ and $n_{\mathrm{d}}=100$ and $s=3$ in eqs 7 and 8 and find the global minimum for the $n=5-7,10-16$, and 18-19 clusters starting from arbitrary structures not near the global minimum. All minimizations were performed using a truncated Newton algorithm ${ }^{49}$ with an $r m s$ gradient convergence criterion of $0.0001 \mathrm{kcal} / \mathrm{mol} / \AA$. We note that our implementation of the DEM protocol succeeds in finding the global minimum for the $n=12$ and 19 clusters in contrast to the work of Kostrowicki et al.

A PS-NMLS method was used in an attempt to find the global minimum for the $n=5-39$ and $n=55$ clusters. For the $n=$ 
8 cluster the global minimum energy in Lennard-Jones units is -19.8222 and the first excited state, a dodecahedron of triangle faces, has an energy of -19.7649 LJ units. Both the DEM and PS-NMLS methods recover the first excited state instead of the global minimum. For $n=9$ and $n=17$ we found the global minimum using a PS-NMLS method with the five largest eigenmodes in $\Gamma_{\mathrm{vc}}$ as search directions. Local searches in $\Gamma_{\mathrm{vc}}$ were done for all $t<5.0$ during the reversal.

Clusters of size $n=38,75-77$, and 102-104 are particularly challenging problems for global optimization due to the "multiple funnel" structure of the underlying PES. ${ }^{56}$ Multiple funnels refer to potential energy surfaces with multiple similar low-energy basins and very different conformations. For the $n$ $=38$ case the fcc truncated octahedron is the global minimum with an energy of -173.9284 Lennard-Jones (LJ) units. ${ }^{57}$ PES deformation schemes such as the distance scaling method of Pillardy and Piela ${ }^{57}$ and the "basin hopping" algorithm of Doye and Wales ${ }^{11}$ succeed in finding the global minimum for $n=$ 38. The distance scaling and basin hopping methods generate smoother potential energy surfaces for improved conformational searching. The $n=38$ problem is easy for our version of potential smoothing without local search. The global minimum structure derived from fcc symmetry can be found from completely random starting structures. For some other problems, particularly $n=31,34$, and 37 we were unable to find the global minimum using PS-NMLS.

For the $n=55$ case DEM does not find the global minimum from arbitrary starting structures. This is because at $t=t_{\mathrm{d}}$ it is not possible to obtain a unique structure irrespective of the gradient convergence criterion chosen for the truncated Newton optimization. It is possible that nonunique conformations at large deformations are a consequence of errors in numerical precison. We applied a PS-NMLS protocol to several different starting structures and a small set of search directions along the Cartesian eigenvectors and always succeeded in finding the global minimum. The $n=13,55$ clusters possess a high degree of symmetry because of their perfect Mackay icosahedral structures. For all clusters, the results reported above can be recovered from completely random starting conformations. Results from our application of DEM and PS-NMLS protocols are summarized in Table 2.

2. Oligopeptides in $\boldsymbol{\Gamma}_{\mathbf{v c}}$. DEM and PS-NMLS were applied to $N$-acetyl-Ala-Ala- $N^{\prime}$-methylamide in $\Gamma_{\mathrm{vc}}$. We first exhaustively enumerated all the minima on the undeformed DOPLS surface. This was done by combining an extensive grid search around minimum energy regions to a series of truncated Newton conjugate gradient minimizations. We used a gradient convergence criterion of $0.0001 \mathrm{kcal} / \mathrm{mol} / \AA$. Minimum energy regions for the grid search were chosen based on the work of Zimmerman et al. ${ }^{58}$ The combined grid search procedure finds a total of 142 unique minima. The four lowest minima including the global minimum have energies of $V_{\text {global }}=-84.1906 \mathrm{kcal} /$ $\mathrm{mol}, V_{2}=-83.9167 \mathrm{kcal} / \mathrm{mol}, V_{3}=-81.9962 \mathrm{kcal} / \mathrm{mol}$, and $V_{4}=-81.9509 \mathrm{kcal} / \mathrm{mol}$.

We characterized the smoothing of the PES by following each of the 142 minima as a function of increasing $t$. We used values of $s=3, t_{\mathrm{d}}=55.0$, and $n_{\mathrm{d}}=400$ in eq 7. On the $t=55.0$ surface only a single minimum remains. We observe that the number of minima reduces from 142 at $t=0$ to 1 for $t=55$ through a series of mergers that are a consequence of diminishing barriers between minima. Exhaustive enumeration allows complete characterization of the smoothing process and evaluation of the requirements for finding the global minimum. An
TABLE 2: Results for PS-NMLS and DEM and Energy Minimizations Applied to Clusters of Argon Atoms ${ }^{a}$

\begin{tabular}{|c|c|c|c|}
\hline $\begin{array}{l}\text { cluster } \\
\text { size } n\end{array}$ & $\begin{array}{l}\text { global } \\
\text { minimum }\end{array}$ & $\begin{array}{l}\text { PS-NMLS } \\
\text { minimum }\end{array}$ & $\begin{array}{l}\text { no. of } \Gamma_{\mathrm{vc}} \\
\text { search } \\
\text { directions } \\
\text { used in } \\
\text { PS-NMLS }\end{array}$ \\
\hline 5 & -9.1039 & -9.1039 & 0 \\
\hline 6 & -12.7121 & -12.7121 & 0 \\
\hline 7 & -16.5054 & -16.5054 & 0 \\
\hline 8 & -19.8222 & -19.7649 & 24 \\
\hline 9 & -24.1134 & -23.2698 & 5 \\
\hline 10 & -28.4225 & -28.4225 & 0 \\
\hline 11 & -32.7660 & -32.7660 & 0 \\
\hline 12 & -37.9676 & -37.9676 & 0 \\
\hline 13 & -44.3268 & -44.3268 & 0 \\
\hline 14 & -47.8452 & -47.8452 & 0 \\
\hline 15 & -52.3226 & -52.3226 & 0 \\
\hline 16 & -56.8157 & -56.8157 & 0 \\
\hline 17 & -61.3180 & -61.3180 & 5 \\
\hline 18 & -66.5309 & -66.5309 & 0 \\
\hline 19 & -72.6598 & -72.6598 & 0 \\
\hline 20 & -77.1770 & -77.1770 & 10 \\
\hline 21 & -81.6846 & -81.6846 & 10 \\
\hline 22 & -86.8098 & -81.8098 & 15 \\
\hline 23 & -92.8445 & -92.8445 & 23 \\
\hline 24 & -97.3488 & -97.3488 & 15 \\
\hline 25 & -102.3727 & -102.3727 & 15 \\
\hline 26 & -108.3156 & -108.3156 & 15 \\
\hline 27 & -112.8736 & -112.8736 & 15 \\
\hline 28 & -117.8224 & -117.8224 & 25 \\
\hline 29 & -123.5874 & -123.5874 & 29 \\
\hline 30 & -128.2866 & -128.2866 & 55 \\
\hline 31 & -133.5864 & -133.1836 & 87 \\
\hline 32 & -139.6355 & -139.6355 & 21 \\
\hline 33 & -144.8427 & -144.8427 & 20 \\
\hline 34 & -150.0445 & -149.6721 & 96 \\
\hline 35 & -155.7566 & -155.7566 & 44 \\
\hline 36 & -161.8254 & -161.8254 & 23 \\
\hline 37 & -167.0338 & -166.6315 & 105 \\
\hline 38 & -173.9284 & -173.9284 & 0 \\
\hline 39 & -180.0332 & -180.0332 & 23 \\
\hline 55 & -279.2485 & -279.2485 & 10 \\
\hline
\end{tabular}

${ }^{a}$ In the table $n$ denotes the size of the cluster studied. If the number of search directions for PS-NMLS is zero, then a straight DEM protocol succeeds at finding the global minimum. All energies are in LennardJones (LJ) units.

analysis of smoothing applied to the fully characterized PES of $N$-acetyl-Ala-Ala- $N^{\prime}$-methylamide will be reported elsewhere. ${ }^{59}$

An energy crossing between minimum 4 and the global minimum occurs at $t=0.1372$, i.e., $V_{4}<V_{\text {global }}$ for all values of $t \geq 0.1372$. The smoothing process projects out a catchment region related to minimum 4 for large $t$, and a DEM reversing schedule converges to minimum 004 at $t=0$, i.e., $V_{4}=$ $-81.9509 \mathrm{kcal} / \mathrm{mol}$. A NMLS protocol using a search along five large torsional eigenmodes corrects post facto the consequence of the crossing at $t=0.1302$ and converges to the global minimum. This example illustrates how a local search method can correct errors due crossings during the smoothing process.

We tested the ability of a PS-TSBS protocol to find the global minimum for $N$-acetyl-Ala-Ala- $N^{\prime}$-methylamide. In the TSBS method the first stage is the location of a nearby saddle point via the $\mathbf{G}$-vector method described earlier. This is followed by perturbation away from the saddle point along the eigenvectors corresponding to the negative eigenvalue in order to reach the adjacent minimum.

We tuned the TSBS searches to find the global minimum by correcting for some of the problems inherent to locating transition states. A point for energy minimization is found after 
TABLE 3: Ideal Geometries Used for Constructing Capped Polyalanine Chains Using DOPLS Definitions of Atom Types for Varying Lengths in $\boldsymbol{\Gamma}_{\mathrm{vt}}{ }^{a}$

\begin{tabular}{lclc}
\hline \multicolumn{1}{c}{ bonds } & bond lengths $(\AA)$ & \multicolumn{1}{c}{ angles } & bond angles $(\mathrm{deg})$ \\
\hline $\mathrm{C}-\mathrm{CH}_{3}$ & 1.51 & $\mathrm{CH}_{3}-\mathrm{C}^{\prime}-\mathrm{O}$ & 122.5 \\
$\mathrm{C}^{\prime}=\mathrm{O}$ & 1.22 & $\mathrm{CH}_{3}-\mathrm{C}^{\prime}-\mathrm{N}$ & 114.4 \\
$\mathrm{~N}-\mathrm{C}^{\prime}$ & 1.34 & $\mathrm{C}^{\prime}-\mathrm{N}-\mathrm{C}_{\alpha} \mathrm{H}$ & 121.0 \\
$\mathrm{C}_{\alpha} \mathrm{H}-\mathrm{N}$ & 1.46 & $\mathrm{~N}-\mathrm{C} \alpha \mathrm{H}-\mathrm{C}^{\prime}$ & 111.0 \\
$\mathrm{C}^{\prime}-\mathrm{C}_{\alpha} \mathrm{H}$ & 1.51 & $\mathrm{C}_{\alpha} \mathrm{H}-\mathrm{C}^{\prime}-\mathrm{O}$ & 122.5 \\
$\mathrm{~N}-\mathrm{HN}$ & 1.02 & $\mathrm{C}^{\prime}-\mathrm{N}-\mathrm{H}$ & 118.0 \\
$\mathrm{C}-{ }^{\prime} \mathrm{C}_{\alpha} \mathrm{H}$ & 1.54 & $\mathrm{~N}-\mathrm{C}_{\alpha} \mathrm{H}-\mathrm{CH}_{3}$ & 109.5 \\
$\mathrm{C}_{\alpha} \mathrm{H}-\mathrm{CH}_{3}$ & 1.54 & $\mathrm{C}_{\alpha} \mathrm{H}-\mathrm{C}^{\prime}-\mathrm{N}$ & 112.7 \\
& & $\mathrm{C}^{\prime}-\mathrm{N}-\mathrm{CH}_{3}$ & 121.0
\end{tabular}

${ }^{a}$ On this manifold the values of $\omega$ are kept fixed at $180^{\circ}$.

an NMLS type walk away from the saddle point region indicates a turn toward a minimum. We found the global minimum using this method in multiple independent runs starting from random locations on the network of 142 minima. Because transition states were located using random perturbations away from local minima, deviations from a DEM reversal were found at different values of $t$ for each run.

Despite the success in reproducibly finding the global minimum, the current TSBS method is unlikely to work for larger systems. The main disadvantage of the current implementation of PS-TSBS is that the search process becomes nonlocal; i.e., the system is moved farther away from the catchment region of interest than is necessary. However, the concept of moving the system over transition states remains attractive and alternate methods to restrict the climb along the G-vector modulated by information about the appropriate contour of tangency may be a useful way of refining TSBS methods. For the rest of this work we use only the NMLS class of local search enhancements to DEM.

3. $\mathbf{C H}_{3} \mathbf{C O}-(\mathrm{L}-\mathrm{Ala})_{n}-\mathrm{NHCH}_{3}$ in $\boldsymbol{\Gamma}_{\mathrm{vt}}$ and $\boldsymbol{\Gamma}_{\mathrm{vc}}$. Nakamura et al. ${ }^{38}$ have studied DEM conformational energy minimization of a capped 9-residue polyalanine chain using a modification of an AMBER 4.0 force field. They showed that a DEM protocol fails to find the global minimum, believed to be an $\alpha$-helix, while a "two-stage" method recovers a structure slightly lower in energy than the $\alpha$-helix that is very similar to the canonical $\alpha$-helix, differing only in a bifurcated "capping" hydrogen bond at the C-terminus.

We analyzed varying lengths of capped polyalanine sequences $\mathrm{CH}_{3} \mathrm{CO}-(\mathrm{L}-\mathrm{Ala})_{n}-\mathrm{NHCH}_{3}$ in $\Gamma_{\mathrm{vt}}$. The bonds and angles are invariant in all of these calculations and were based on idealized peptide values shown in Table 3. Because the torsional barrier for deviation away from $\omega=180^{\circ}$ is large, the $\omega$ angle was kept fixed at $180^{\circ}$, the trans conformation of the peptide bond. It can be shown that $t=t_{\mathrm{d}}=10$ is a sufficiently large extent of smoothing for random starting structures to converge to the same energy and conformation. Through systematic trials we found $s=5$ to be appropriate for smoothing in $\Gamma_{\mathrm{vt}}$. Values of $n_{\mathrm{d}}$ were varied based on the size of the problem. As the number of residues, $n$, is increased beyond 7 for $\mathrm{CH}_{3} \mathrm{CO}-(\mathrm{L}-\mathrm{Ala})_{n}-$ $\mathrm{NHCH}_{3}$ in $\Gamma_{\mathrm{vt}}$ the number of points $n_{\mathrm{d}}$ between $t=t_{\mathrm{d}}$ and $t=$ 0 is increased. If the decrease in smoothing level $\Delta t$ is sufficiently small during the reversing schedule, an NMLS protocol is reduced to making a binary decisions in multidimensional conformational space. If $\Delta t$ is not small enough, then a NMLS protocol may require multiple iterations to converge to the best alternate minimum. For larger values of $\Delta t$, an alternate minimum may be too far from the local minimum to be found by a NMLS protocol at the current level of smoothing.
TABLE 4: Summary of Results from Application of PS-NMLS to Varying Lengths of $\mathrm{CH}_{3} \mathrm{CO}-(\mathrm{L}-\mathrm{Ala})_{n}-\mathrm{NHCH}_{3}$ Sequences in $\boldsymbol{\Gamma}_{\mathrm{vt}}{ }^{a}$

\begin{tabular}{rccclc}
\hline & $\begin{array}{c}\text { no. of } \\
\text { flexible } \\
\text { torsions } \\
n\end{array}(\phi, \psi)$ & $\begin{array}{c}\text { DEM } \\
\text { energy } \\
(\mathrm{kcal} / \mathrm{mol})\end{array}$ & $\begin{array}{c}\text { PS-NMLS } \\
\text { energy } \\
(\mathrm{kcal} / \mathrm{mol})\end{array}$ & $\begin{array}{c}\text { PS-NMLS } \\
\text { structure } \\
\text { type }\end{array}$ & $\begin{array}{c}\text { energy of } \\
\text { canonical } \\
\alpha \text {-helix in } \\
\Gamma_{\mathrm{vt}}\end{array}$ \\
\hline 5 & 10 & -179.4897 & -179.4897 & $\gamma$-turn & -178.2146 \\
6 & 12 & -212.5233 & -217.4073 & $\beta$-hairpin & -215.5294 \\
7 & 14 & -245.5841 & -253.8166 & $\alpha^{\prime}$-helix & -253.3204 \\
8 & 16 & -278.6389 & -293.4585 & $\beta$-hairpin & -291.4040 \\
9 & 18 & -311.7031 & -330.0448 & $\alpha^{\prime}$-helix & -329.7243 \\
10 & 20 & -344.7672 & -368.4315 & $\alpha^{\prime}$-helix & -368.1972 \\
11 & 22 & -377.8342 & -406.9659 & $\alpha^{\prime}$-helix & -406.7670 \\
12 & 24 & -410.9029 & -445.5773 & $\alpha^{\prime}$-helix & -445.4150
\end{tabular}

${ }^{a}$ In $\Gamma_{\mathrm{vt}}$ the bonds and angles remain fixed and the energy minimizations find the lowest or global minimum on the manifold of fixed bond and angles.

Results from application of PS-NMLS to chains of $\mathrm{CH}_{3} \mathrm{CO}-$ (L-Ala) $)_{n}-\mathrm{NHCH}_{3}$ in $\Gamma_{\mathrm{vt}}$ for $n=5-12$ are described in Table 4. For all of the chains DEM obtains a $\gamma$-turn structure. For $n=5$ the $\gamma$-turn is the DOPLS global energy minimum. For $n=6$ and 8 , we find $\beta$-hairpin structures with nonclassical reverse turns to be the lowest energy structures. For $n=7$ and $n=9-12$, the PS-NMLS method recovers structures very similar to, and slightly lower in energy than, canonical $\alpha$-helices. Differences between the two types of structures are in the C-termini where the $N$-methyl amide hydrogen forms a bifurcated hydrogen bond with carbonyl oxygens from the residues $n-1$ and $n-2$. We refer to such structures as $\alpha^{\prime}$-helices. Values for the torsional $\phi$ and $\psi$ are angles are similar to those of canonical $\alpha$-helices.

Each of the PS-NMLS calculations in $\Gamma_{\mathrm{vt}}$ for $\mathrm{CH}_{3} \mathrm{CO}-$ $(\mathrm{L}-\mathrm{Ala})_{n}-\mathrm{NHCH}_{3}$ chains requires approximately a thousand independent minimizations. It is instructive to compare results from our PS-NMLS calculations to a random search that uses the same number of local minimizations. For each of the $\mathrm{CH}_{3} \mathrm{CO}-(\mathrm{L}-\mathrm{Ala})_{n}-\mathrm{NHCH}_{3}$ chains we set the angle $\omega$ to be trans and generated a thousand independent conformations using random values of $\phi$ and $\psi$ between $-180^{\circ}$ and $180^{\circ}$. Each of the 1000 starting conformations were minimized using an optimally conditioned quasi-Newton method without line searches ${ }^{48}$ over $\phi-\psi$ space to an $r m s$ gradient convergence of $0.0001 \mathrm{kcal} / \mathrm{mol} / \mathrm{radian}$. We were unable to find the global minimum for any of the polyalanine chains studied using this random search procedure. The same 1000 randomized starting conformations were used as starting positions for a NMLS local search optimization on the undeformed surface. For $n=5-8$ we found the global minimum approximately $35 \%$ of the time and for $n=9-12$ the success in finding the global minimum ranges from $22-30 \%$. The improved success of NMLS optimizations on the undeformed surface over a random search is an indication that the iterative scheme in the search procedure facilitates improved sampling of conformational space. On average we required six or more iterations of local search to converge to the global minimum. The important virtue of the PS-NMLS over NMLS on the undeformed surface is that for $n$ =5-12 all of the 1000 random conformations merge into a single conformation with the same energy for $t=t_{\mathrm{d}}=10$. This means that the smoothing procedure is completely deterministic and does not require a large number of independent minimizations. Also, as the size of the problem increases, the efficiency of using NMLS on the undeformed surface in finding the global minimum or lower lying minima is greatly reduced. 
TABLE 5: Results of DEM and PS-NMLS Applied to Capped Sequences of Polyalanine, $\mathrm{CH}_{3} \mathrm{CO}-(\mathrm{L}-\mathrm{Ala})_{n}-\mathrm{NHCH}_{3}$ in $\Gamma_{\mathrm{vc}}{ }^{a}$

\begin{tabular}{rccc}
\hline & $\begin{array}{c}\text { DEM } \\
\text { energy } \\
(\mathrm{kcal} / \mathrm{mol})\end{array}$ & $\begin{array}{c}\text { PS-NMLS } \\
\text { energy } \\
(\mathrm{kcal} / \mathrm{mol})\end{array}$ & $\begin{array}{c}\text { canonical } \\
\alpha-h e l i x \text { energy } \\
(\mathrm{kcal} / \mathrm{mol})\end{array}$ \\
\hline 5 & -183.1443 & -197.9965 & -188.3236 \\
6 & -216.9159 & -238.3538 & -227.1757 \\
7 & -250.7094 & -272.9906 & -266.8064 \\
8 & -285.8123 & -314.2375 & -306.8196 \\
9 & -318.3087 & -350.0894 & -346.9622 \\
10 & -352.1148 & -387.0713 & -387.2196 \\
11 & -385.9248 & -423.6528 & -427.5763
\end{tabular}

${ }^{a}$ A schedule of $s=3, n_{\mathrm{d}}=100$, and $t_{\mathrm{d}}=10$ was used in this study.

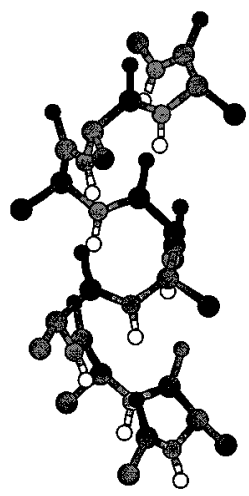

(a)

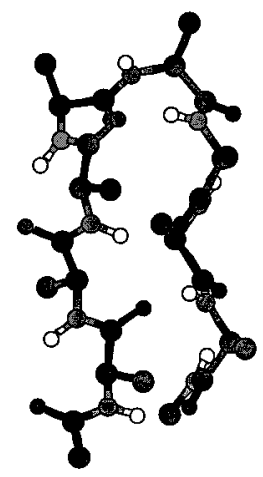

(b)
Figure 1. (a) Lowest energy $\alpha$-helical conformations of $\mathrm{CH}_{3} \mathrm{CO}-$ (L-Ala) $)_{8}-\mathrm{NHCH}_{3}$ in $\Gamma_{\mathrm{vc}}$. (b) The $\beta$-hairpin structure which is 7.4179 $\mathrm{kcal} / \mathrm{mol}$ lower in energy than the canonical $\alpha$-helix shown in (a) and is the structure found using PS-NMLS.

$\boldsymbol{\Gamma}_{\text {vc }}$ Calculations. We studied DEM and PS-NMLS conformational energy minimizations in $\Gamma_{\mathrm{vc}}$ for different lengths of $\mathrm{CH}_{3} \mathrm{CO}-(\mathrm{L}-\mathrm{Ala})_{n}-\mathrm{NHCH}_{3}$ chains. Kostrowicki and Scheraga ${ }^{46}$ have discussed implications of deforming penalty function terms such as harmonic bond length and bond angle restraint terms for DEM smoothing. Because we are not interested in significant rearrangements of covalent geometry, we do not smooth the bond stretch, angle bending, and improper dihedral terms for calculations in $\Gamma_{\mathrm{vc}}$. This is different from $\Gamma_{\mathrm{vt}}$ calculations where bond lengths and bond angles are kept fixed. In $\Gamma_{\mathrm{vc}}$, bonds and angles are allowed to stretch and bend as they would on the undeformed surface. The bonds and angles deform at different levels of smoothing to compensate for longrange effects of nonbonded interactions.

As in $\Gamma_{\mathrm{vt}}$, we set $t_{\mathrm{d}}=10.0$ in all our calculations. We use a fixed schedule of $n_{\mathrm{d}}=100$ and $s=3$ in eqs 7 and 8 for forward smoothing and reversing and study capped polyalanine chains of length $n=5-11$ in vacuo. For all values of $n$, DEM again finds $\gamma$-turn structures. PS-NMLS finds a set of $\beta$-hairpin structures for each of the sequences studied. The $\beta$-hairpin structures found are lower in energy than the $\alpha$-helices in $\Gamma_{\mathrm{vc}}$ for $n=5-9$.

For instance with $n=8$, the $t=0$ DOPLS energy for a local minimum corresponding to the canonical $\alpha$-helix is -306.8196 $\mathrm{kcal} / \mathrm{mol}$. This structure was located using a model-built $\alpha$-helix as the starting conformation and then minimizing to a rms gradient convergence of $0.0001 \mathrm{kcal} / \mathrm{mol} / \mathrm{A}$. A PS-NMLS protocol yields a $\beta$-hairpin structure that is $7.4179 \mathrm{kcal} / \mathrm{mol}$ lower in energy than this $\alpha$-helix. Results of all $\Gamma_{\mathrm{vc}}$ calculations for $n=5-11$ chains of $\mathrm{CH}_{3} \mathrm{CO}-(\mathrm{L}-\mathrm{Ala})_{n}-\mathrm{NHCH}_{3}$ are summarized in Table 5. Figure 1 shows the structures for a $n=8$

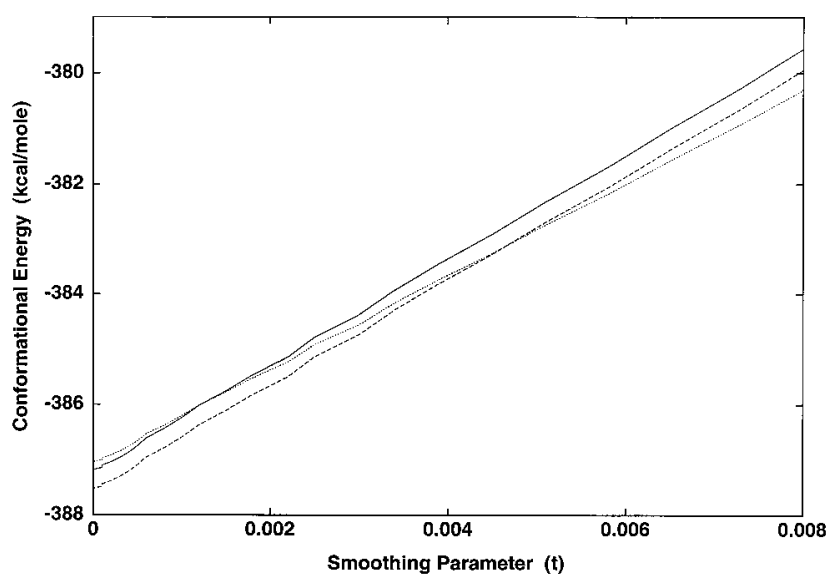

Figure 2. Conformational energies of $\mathrm{CH}_{3} \mathrm{CO}-(\mathrm{L}-\mathrm{Ala})_{10}-\mathrm{NHCH}_{3} \Gamma_{\mathrm{vc}}$ as a function of increasing deformation $t$ for a canonical $\alpha$-helix $(-)$, for an $\alpha^{\prime}$-helix (---), and for the $\beta$-hairpin found from a PS-NMLS protocol $(\cdots)$. On the $t=0.0051$ surface the $\beta$-hairpin is lower in energy than the $\alpha$-helix.

canonical $\alpha$-helix and the lower energy $\beta$-hairpin obtained from a PS-NMLS in $\Gamma_{\mathrm{vc}}$.

In direct analogy to the $\Gamma_{\mathrm{vt}}$ calculation we expected to see $\alpha$-helices as the result of PS-NMLS calculations for longer sequences. For $n=10$ and 11, an PS-NMLS method does not find structures lower in energy than the $\alpha$-helix, but instead finds $\beta$-hairpins that are higher in energy than the $\alpha$-helix. For longer chains we performed exhaustive local searches along all the torsional eigenmodes and found the higher energy $\beta$-hairpins. We analyzed the reasons for this result by enumerating structures sampled during local searches. For $n=10$ there are three unique minima near the global minimum on the DOPLS surface in $\Gamma_{\mathrm{vc}}$, a $\beta$-hairpin $\left(V_{\beta}=-387.0713 \mathrm{kcal} / \mathrm{mol}\right)$, the canonical $\alpha$-helix $\left(V_{\alpha}=-387.2196 \mathrm{kcal} / \mathrm{mol}\right)$, and an $\alpha^{\prime}$-helix $\left(V_{\alpha^{\prime}}=\right.$ $-387.5620 \mathrm{kcal} / \mathrm{mol}$ ) which is the global minimum. We studied the forward smoothing of these three low-energy structures. For values of $t$ between 0.0051 and 0.1826 the global minimum is in the catchment region of the $\beta$-hairpin. A comparison of conformational energies as a function of $t$ for forward smoothing is shown in Figure 2. The $\alpha^{\prime}$-helix becomes higher in energy than the $\beta$-hairpin for $t$ of 0.0051 and greater. For all $t>0.1826$ the global minimum is the $\gamma$-turn basin. The PS-NMLS converges to the global minimum up to the $t=0.0051$ surface which is a $\beta$-hairpin. At the next smaller $t$ value in this particular protocol, $t=0.0045$, the $\alpha^{\prime}$-helix is lower in energy than the $\beta$-hairpin. PS-NMLS fails to recognize the crossing of energies for $0.0045<t<0.0051$ when the $\alpha^{\prime}$-helix becomes lower in energy than the $\beta$-hairpin.

We used the NMLS search strategy to enumerate all local minima sampled from the $\beta$-hairpin and $\alpha^{\prime}$-helix minima along all of the search directions corresponding to the 20 pairs of $\phi-\psi$ angles at $t=0.0045$ and $t=0.0051$. On the $t=0.0045$ surface, the $\alpha^{\prime}$-helix is lower in energy than the $\beta$-hairpin, $V_{\alpha^{\prime}}=$ $-383.2723 \mathrm{kcal} / \mathrm{mol}$ and $V_{\beta}=-383.2569 \mathrm{kcal} / \mathrm{mol}$. Structures found from a local search were characterized as $\alpha$-helical if the distances $r_{i, i+4}$ between the carbonyl oxygen atoms of residue $i$ and the amide nitrogen atom of residue $i+4$ were between 2.7 and $3.1 \AA$, implying $\alpha$-helical hydrogen bonds. If the structures were such that the distances between the atom pairs $\left(\mathrm{O}_{9}-\mathrm{N}_{2}\right),\left(\mathrm{O}_{7}-\mathrm{N}_{4}\right),\left(\mathrm{O}_{4}-\mathrm{N}_{7}\right)$, and $\left(\mathrm{O}_{2}-\mathrm{N}_{9}\right)$ were between 2.7 and $3.1 \AA$, the structure was deemed a $\beta$-hairpin containing interstrand hydrogen bonds. Of the 23 unique low-energy structures found from a local search out of the $\alpha^{\prime}$-helix local 
TABLE 6: Hydrogen-Bonding Distances for the 23 Unique Local Minimum Energy Structures Sampled in a Local Search out of the $\alpha^{\prime}$-Helical Local Minimum on the $t=0.0045$ Surface $^{a}$

\begin{tabular}{|c|c|c|c|c|c|c|c|c|c|c|c|}
\hline \multirow[b]{2}{*}{ rank } & \multirow[b]{2}{*}{ energy } & \multicolumn{6}{|c|}{$\begin{array}{c}i-i+4 \text {, distances between carbonyl oxygen }(i) \text { and } \\
\text { amide nitrogen }(i+4) \text { atoms if the distances are } \\
>2.7 \AA \text { and }<3.1 \AA\end{array}$} & \multicolumn{4}{|c|}{$\begin{array}{l}i-j \text { distances between carbonyl oxygen }(i) \text { and } \\
\text { amide nitrogen }(j) \text { atoms if the distances are } \\
>2.7 \AA \text { and }<3.1 \AA\end{array}$} \\
\hline & & $1-5$ & $2-6$ & $3-7$ & $4-8$ & $5-9$ & $6-10$ & $9-2$ & $7-4$ & $2-9$ & $4-7$ \\
\hline 1 & -383.2723 & 2.87 & 2.96 & 2.87 & 2.91 & 2.91 & & & & & \\
\hline 2 & -382.9076 & 2.87 & 2.95 & 2.88 & 2.90 & 2.92 & 2.95 & & & & \\
\hline 3 & -381.8493 & 2.92 & 2.90 & 2.94 & 2.90 & 2.91 & & & & & \\
\hline 4 & -380.2025 & 2.87 & 2.95 & 2.89 & 2.93 & 2.80 & & & & & \\
\hline 5 & -379.6051 & 2.87 & 2.98 & 2.89 & & & & & & & \\
\hline 6 & -379.4903 & 2.87 & 2.95 & 2.90 & & & & & & & \\
\hline 7 & -377.3410 & 2.81 & & & & 2.92 & & & & & \\
\hline 8 & -375.4960 & 2.96 & 2.89 & 2.92 & 2.88 & & 3.09 & & & & \\
\hline 9 & -373.6687 & & 2.87 & 2.95 & & & & & & & \\
\hline 10 & -373.082 & & & & & 2.82 & & & & & \\
\hline 11 & -372.6473 & 2.89 & & & & & & & & & \\
\hline 12 & -371.9262 & & 2.98 & 2.79 & & & & & & & \\
\hline 13 & -371.5315 & & & & 2.88 & 3.02 & 2.92 & & & & \\
\hline 14 & -370.9817 & 2.92 & 2.83 & 2.96 & & & & & & & \\
\hline 15 & -369.4312 & & & 2.93 & 2.90 & 3.02 & & & & & \\
\hline 16 & -368.719 & 2.86 & & & & & & & & & \\
\hline 17 & -365.4600 & & & & 2.96 & 2.90 & & & & 3.01 & \\
\hline 18 & -362.7182 & & 2.98 & 2.89 & 2.83 & & & & & & \\
\hline 19 & -362.7182 & & 2.98 & 2.89 & 2.83 & & & & & & \\
\hline 20 & -358.1491 & & & & & & & & & & \\
\hline 21 & -357.0376 & & & & & & & & & & \\
\hline 22 & -353.3007 & & & & & & & & & & \\
\hline 23 & -347.6971 & & & & & & & & & & \\
\hline
\end{tabular}

${ }^{a}$ Columns $3-8$ of the table correspond to the type of hydrogen bonds that classify $\alpha$-helical structures. All the very low energy structures show typical $\alpha$-helical hydrogen-bonding pattern. The number of $\alpha$-helical hydrogen bonds decreases with increase in conformational energy and the higher energy structures sampled in this calculation are random coil conformations. The table reflects two important features of the local search sampling. The $\alpha$-helical basin and the $\beta$-hairpin basin are disjoint sets and the $\alpha$-helical basin is a very narrow deep well reflected in the fewer number of structures sampled, compared to the $\beta$-hairpin.

minimum, almost all show the hydrogen-bonding pattern of an $\alpha$-helix. Results of this search are shown in Table 6. Similarly, a local search out of the $\beta$-hairpin minimum at $t=0.0051$ finds 32 unique local minima including the original $\beta$-hairpin. More than $90 \%$ of the structures show hydrogen-bonding patterns typical of a $\beta$-hairpins; see Table 7 . The lack of overlap in the conformational energies listed in these two tables show that local searches out of the $\alpha^{\prime}$-helical and $\beta$-hairpin basins sample conformationally disjoint regions. Similar results were obtained for calculations on the $t=0.0051$ surface for which the $\beta$-hairpin is lower in energy than the $\alpha^{\prime}$-helix, $V_{\beta}=-382.7399 \mathrm{kcal} / \mathrm{mol}$ versus $V_{\alpha^{\prime}}=-382.6904 \mathrm{kcal} / \mathrm{mol}$.

Three important observations from these local search calculations are as follows: (1) we systematically find more unique local minima out of the $\beta$-hairpin minimum than the $\alpha^{\prime}$-hairpin minimum, suggesting that the $\beta$-hairpin basin may be broader than the $\alpha$-helical basin; (2) the hydrogen-bonding pattern suggests that the two sets of conformations sampled from a local search out of an $\alpha$-helical minimum and $\beta$-hairpin minimum are disjoint and are separated by a large barrier; (3) local search cannot surmount the barrier between the narrow $\alpha^{\prime}$-helix and broad $\beta$-hairpin regions for $t<0.0051$ and stays within the catchment region of the $\beta$-hairpin all the way back to the $t=$ 0 DOPLS surface.

To test the effect of force field parametrization we repeated calculations for the $n=5$ and 8 capped polyalanine sequences in $\Gamma_{\mathrm{vc}}$ using a deformable version of the CHARMM22 force field in place of DOPLS. The only modification to the CHARMM22 energy functions was made in the substitution of a Gaussian approximation to the 12-6 Lennard-Jones function for van der Waals interactions. An energy minimized canonical $\alpha$-helix for $n=5$ using modified CHARMM22 has an undeformed surface energy of $85.4704 \mathrm{kcal} / \mathrm{mol}$. A PS-NMLS protocol finds a structure that is not an $\alpha$-helix, but which is lower in energy. The undeformed surface energy for this structure is $73.7718 \mathrm{kcal} / \mathrm{mol}$. The DOPLS surface minimum energy structure shows an $r m s$-carbon deviation of $1.8268 \AA$ from the undeformed CHARMM surface minimum energy structure. The $(\phi, \psi)$ angles for the DOPLS and CHARMM22 structures are quite similar. For $n=8$ the CHARMM22 $\alpha$-helix energy is $141.8306 \mathrm{kcal} / \mathrm{mol}$. PS-NMLS finds a $\beta$-hairpin structure with a lower energy of $133.5861 \mathrm{kcal} / \mathrm{mol}$. The DOPLS surface minimum energy $\beta$-hairpin for $n=8$ shows a deviation of $1.2175 \AA$ in $\alpha$-carbon atom positions from the undeformed CHARMM surface minimum energy $\beta$-hairpin. this calculation demonstrates that PS-NMLS protocols using both DOPLS and CHARMM22 force fields give qualitatively similar results for $\Gamma_{\mathrm{vc}}$ calculations on small polyalanine sequences. We also performed a $\Gamma_{\mathrm{vt}}$ calculation using modified CHARMM22 functions for an $n=12$ capped polyalanine sequence. The PSNMLS method recovers the $\alpha$-helix as the global minimum on the manifold of fixed idealized CHARMM22 bonds lengths and bond angles.

The efficiency of the PS-NMLS method was compared to a random search using approximately the same number of local minimizations, and to NMLS on the undeformed surface from random starting conformations. Neither of these methods succeeds in finding the global minimum for $n=5-9$. For $n$ $=10$ the lowest energy conformer found using the random search method is $17.2 \mathrm{kcal} / \mathrm{mol}$ higher in energy than the PSNMLS $\beta$-hairpin, and the lowest energy conformer found using the $t=0$ surface NMLS is $6.34 \mathrm{kcal} / \mathrm{mol}$ higher in energy than the PS-NMLS structure.

4. Cycloheptadecane in $\boldsymbol{\Gamma}_{\mathbf{v c}}$. One of the better known benchmark problems for conformational search is the determination of low-energy conformations of the highly flexible 
TABLE 7: Hydrogen-Bonding Distances for 32 Unique Local Minima Sampled in a Local Search out of the $\beta$-Hairpin Local Minimum on the $t=0.0045$ Surface $^{a}$

\begin{tabular}{|c|c|c|c|c|c|c|c|c|c|c|c|}
\hline \multirow[b]{2}{*}{ rank } & \multirow[b]{2}{*}{ energy } & \multicolumn{6}{|c|}{$\begin{array}{l}-i+4 \text {, distances between carbonyl oxygen }(i) \text { and } \\
\text { amide nitrogen }(i+4) \text { atoms if the distances are } \\
>2.7 \AA \text { and }<3.1 \AA\end{array}$} & \multicolumn{4}{|c|}{$\begin{array}{l}i-j \text { distances between carbonyl oxygen }(i) \text { and } \\
\text { amide nitrogen }(j) \text { atoms if the distances are } \\
>2.7 \AA \text { and }<3.1 \AA\end{array}$} \\
\hline & & $1-5$ & $2-6$ & $3-7$ & $4-8$ & $5-9$ & $6-10$ & $9-2$ & $7-4$ & $2-9$ & $4-7$ \\
\hline 1 & -383.2569 & & & & & & & 2.82 & 2.86 & 2.88 & 2.93 \\
\hline 1 & -383.2569 & & & & & & & 2.82 & 2.86 & 2.88 & 2.93 \\
\hline 2 & -381.8750 & & & & & & & 2.76 & 2.82 & 2.87 & 2.93 \\
\hline 3 & -380.7871 & & & & & & & & 2.88 & 2.98 & 2.90 \\
\hline 4 & -378.7298 & & & & & & & & 2.86 & 2.97 & 2.91 \\
\hline 5 & -377.0667 & & & & & & & 3.00 & 2.90 & 2.80 & $2.92^{\circ}$ \\
\hline 6 & -376.5693 & & & & & & & & 2.79 & 2.81 & 2.94 \\
\hline 7 & -376.3553 & & & & & & & & 2.85 & & 2.94 \\
\hline 8 & -376.2071 & & & & & & & 2.85 & 2.85 & 2.86 & 2.93 \\
\hline 9 & -375.9423 & & & & & & & & 2.78 & 2.84 & 2.95 \\
\hline 10 & -375.5653 & & & & & & & & 2.80 & 2.82 & 2.93 \\
\hline 11 & -375.0034 & & & & & & & 2.84 & 2.90 & 2.88 & 2.92 \\
\hline 12 & -374.3673 & & & & & & & & 2.83 & 2.85 & 2.92 \\
\hline 13 & -373.6144 & & & & & & 2.83 & & & & \\
\hline 14 & -372.4078 & & & & & & & & & & 2.86 \\
\hline 15 & -372.2050 & & & & & & & 2.77 & 2.95 & 2.86 & 3.02 \\
\hline 16 & -372.1612 & & & & & & & & 2.84 & & 2.90 \\
\hline 17 & -370.1621 & & & & & & & 2.89 & & & \\
\hline 18 & -370.0502 & & & & & & & & 2.85 & 3.02 & 2.91 \\
\hline 19 & -369.6184 & & & & & & & & & & \\
\hline 20 & -368.6573 & & & & & & & 2.82 & 2.88 & & 2.95 \\
\hline 21 & -367.5699 & & & 3.02 & & 2.81 & & & & & \\
\hline 22 & -367.1853 & & & & & & & 2.83 & & & \\
\hline 23 & -366.6323 & & & & & & & 2.88 & & & 2.79 \\
\hline 24 & -365.6136 & & & & & & & 2.81 & 2.85 & 2.87 & 2.92 \\
\hline 25 & -365.5884 & & & & & & & & & & \\
\hline 26 & -365.0771 & & & & & & & & 2.82 & 2.77 & 2.92 \\
\hline 27 & -365.0771 & & & & & & & & 2.82 & 2.77 & 2.92 \\
\hline 28 & -361.5149 & & & & & & & 2.82 & & & \\
\hline 29 & -360.0044 & & & & & & & & & & 2.89 \\
\hline 30 & -359.0971 & & & & 2.93 & & & & & & \\
\hline 31 & -359.0971 & & & & 2.93 & & & & & & \\
\hline 32 & -352.6797 & & & & & & & 2.95 & & 2.87 & 2.99 \\
\hline
\end{tabular}

${ }^{a}$ The last four columns of the table correspond to hydrogen bonds for a $\beta$-hairpin. All low-energy structures sampled from the $\beta$-hairpin local minimum show hydrogen-bonding patterns typical of $\beta$-hairpins. Higher energy structures have fewer $\beta$-hairpin hydrogen bonds and in some cases show a few $\alpha$-helical hydrogen bonds.

cycloheptadecane. ${ }^{60}$ This system continues to attract attention and serve as a test for newly developed search methods. ${ }^{61}$ While not a particularly large molecule, cycloheptadecane presents a difficult challenge due to its great flexibility and the close energy spacing of the lower lying minima. Extensive analysis via a variety of search methods has located exactly 263 minima on the MM2 energy surface within $3.0 \mathrm{kcal} / \mathrm{mol}$ of the purported global minimum. Since the full spectrum of energy minima for this molecule has not been described in the literature, we undertook its generation. We used an iterative NMLS protocol with the MM2 energy function surface to sample the PES of cycloheptadecane. A local search was carried out from every unique minimum found on the PES. All symmetry-distinct minima found in this manner were added to the existing map and the procedure was repeated until self-consistent convergence. The structures found are unique minima to an $r m s$ gradient per atom of $0.00001 \mathrm{kcal} / \mathrm{mol}$ on the MM2 potential energy surface ${ }^{42}$ traditionally used in studies of this system. Using the iterative NMLS scheme to hop between minima we found 20469 unique minimum energy structures with an MM2 energy distribution as shown in Figure 3a. Even with the use of an efficient truncated Newton minimization method, ${ }^{49}$ generation of the full distribution required about 13 days of $\mathrm{CPU}$ time on a $250 \mathrm{MHz}$ DEC Alpha workstation. The global minimum has an MM2 energy of $19.0680 \mathrm{kcal} / \mathrm{mol}$. A second minimum lies only $0.01 \mathrm{kcal} / \mathrm{mol}$ above the global minimum and has an MM2 energy of $19.0774 \mathrm{kcal} / \mathrm{mol}$. These two structures are separated by about $0.4 \mathrm{kcal} / \mathrm{mol}$ from the third best and subsequent structures. The low-energy tail of the full distribution is presented in Figure $3 b$.

Application of the PS-NMLS algorithm to cycloheptadecane in $\Gamma_{\mathrm{vc}}$ succeeds in finding the second lowest minimum. We used a maximum deformation of $t_{\mathrm{d}}=25.0$ at which point only one minimum remains on the smoothed surface. Variations in the reversal protocol $\left(n_{\mathrm{d}}=100-150\right.$, and $s=2$ or 3 ) coupled with variation in the number of modes searched during NMLS (values from 3 to 16) also result in the procedure finding the second lowest minimum.

The PS-NMLS protocol fails to find the global minimum for the same reasons that it fails for longer polyalanine chains in $\Gamma_{\mathrm{vc}}$. In Table 8 we summarize the evolution of minima on the PES as a function of smoothing. There is a crossing of the relative energies of the global minimum and the second lowest minimum for $t \approx 0.0061$. If the PS-NMLS is to successfully converge to the global minimum, the protocol would have to be able to sample the global minimum for values of $t<0.0061$. Smoothed surfaces at very small $t$ where the global minimum is favored closely resemble the original undeformed surface. If the second lowest minimum and the global minimum are in widely separated regions of conformational space, a local search will not be able to sample the global minimum. Further extensions of PS-NMLS that should be able to find several of the lowest energy structures of cycloheptadecane, including the global minimum, are discussed below. Figure 4 shows the 

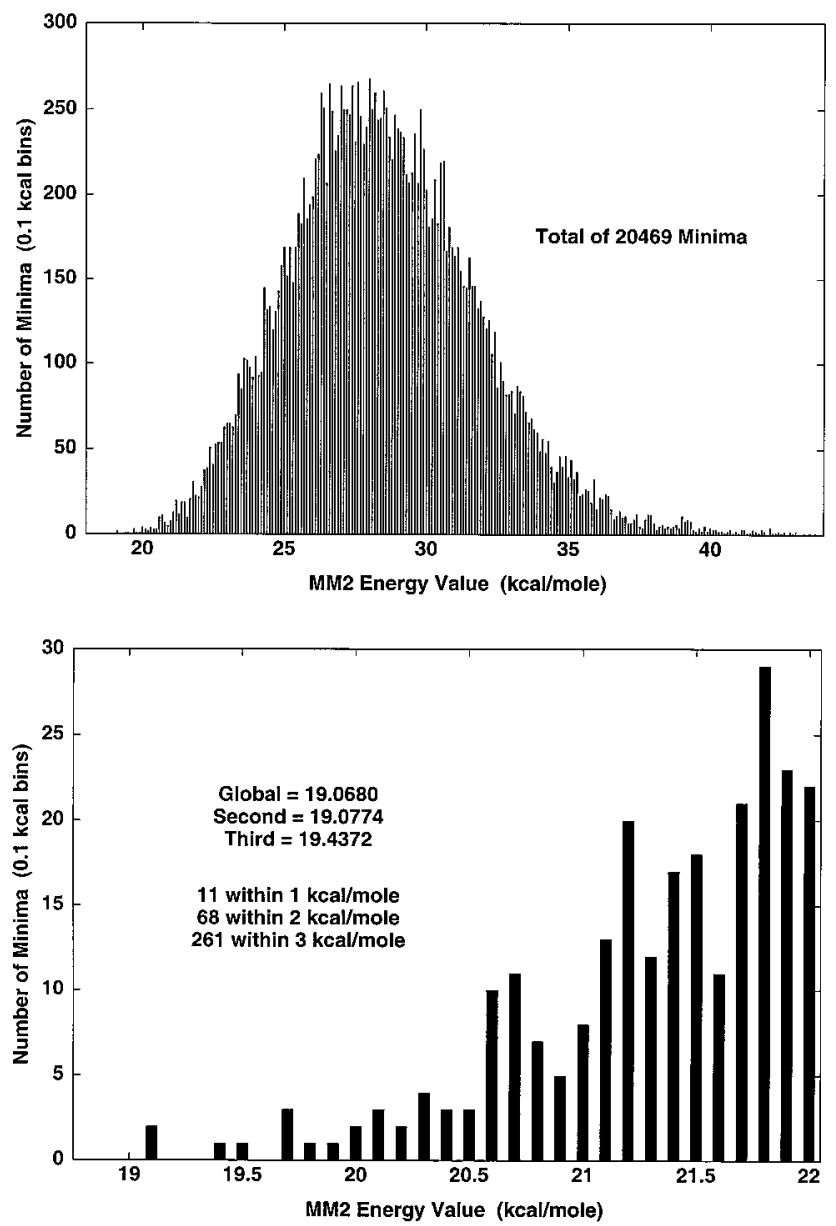

Figure 3. (a, top) Energy distribution of the 20.469 unique minima for cycloheptadecane located using a self-consistent NMLS-based search to scan the complete potential energy surface. The number of minima found in each $0.1 \mathrm{kcal} / \mathrm{mol}$ energy bin is plotted as a function of increasing MM2 energy value. (b, bottom) Low-energy tail of (a) showing the distribution of minima with MM2 energy values less than $3 \mathrm{kcal} / \mathrm{mol}$ above the global minimum. The search procedure used to generate both panels (a) and (b) found 11 minima within $1 \mathrm{kcal} / \mathrm{mol}$ of the global minimum, 68 minima within $2 \mathrm{kcal} / \mathrm{mol}$, and $261 \mathrm{minima}$ within $3 \mathrm{kcal} / \mathrm{mol}$.

global minimum for cycloheptadecane and the second lowest energy structure located by the current PS-NMLS.

The cycloheptadecane problem illustrates some of the advantages of the PS-NMLS method for global optimization. It has been estimated that an extensive search procedure requires approximately 10000 independent minimizations before converging to the global minimum and locating the set of lowlying minima. ${ }^{61}$ A typical PS-NMLS run for cycloheptadecane with $n_{\mathrm{d}}=100, s=3$, and local search along the three highest torsional modes finds the second lowest minimum in less than $1 \mathrm{~h}$ of CPU time on the computer cited above and requires only 440 energy minimizations. This number can perhaps be reduced somewhat further by a coarse reversing schedule (reducing $n_{\mathrm{d}}$ with $s=2$ ) while still yielding the same result as more exhaustive searching. The large reduction in the number of independent minimizations compared with other search techniques derives from the improved sampling on smooth surfaces.

We calibrated the NMLS procedure on the undeformed surface for cycloheptadecane using an iterative NMLS optimization scheme starting from 500 independent randomized conformations. Each self-consistent run of NMLS on the undeformed surface requires approximately 200 minimizations. Only one of the 500 NMLS runs finds the second lowest minimum,
TABLE 8: Evolution of the Lowest 15 Minima of Cycloheptadecane as a Function of Increased Smoothing ${ }^{a}$

\begin{tabular}{|c|c|c|c|c|c|}
\hline rank & $\begin{array}{c}\text { MM2 } \\
\text { energy } \\
(\mathrm{kcal} / \mathrm{mol})\end{array}$ & $\begin{array}{c}t=0 \\
\text { energy } \\
(\mathrm{kcal} / \mathrm{mol})\end{array}$ & $\begin{array}{c}t=0.001 \\
\text { energy } \\
(\mathrm{kcal} / \mathrm{mol})\end{array}$ & $\begin{array}{c}t=0.01 \\
\text { energy } \\
(\mathrm{kcal} / \mathrm{mol})\end{array}$ & $\begin{array}{c}t=0.1 \\
\text { energy } \\
(\mathrm{kcal} / \mathrm{mol})\end{array}$ \\
\hline 1 & & 196627 & 61 & 127 & 10 \\
\hline 2 & & 1 & & & 437 \\
\hline 3 & 1 & 20. & & & 972 \\
\hline 4 & & $20 .($ & & & 716 \\
\hline 5 & & 0. & 20 & 32 & 382 \\
\hline 6 & .1292 & 20.3 & 21. & 476 & 107.7838 \\
\hline 7 & .7328 & 20.3 & $21 .($ & 27.4308 & 2037 \\
\hline 8 & & 20.4 & 75 & 27.4956 & 107.1938 \\
\hline 9 & & & & & 107.4179 \\
\hline 10 & & & & & 107.3900 \\
\hline 11 & & & & & 2206 \\
\hline 12 & & & & & 181 \\
\hline 13 & 4 & 20.7 & 21 & 27.8163 & 107.4686 \\
\hline 14 & 1470 & 20.7 & 21. & 27.8836 & 107.8315 \\
\hline 15 & 20.2011 & 20.8072 & 21.5034 & 27.9811 & 108.3395 \\
\hline
\end{tabular}

${ }^{a}$ Column 2 shows the MM2 energies for the lowest energy conformers found using an extensive NMLS-based search technique. We found 261 unique minima within $3 \mathrm{kcal} / \mathrm{mol}$ of the global minimum. A smoothable variant of the MM2 PES which replaces the Buckingham potential with a 2-Gaussian approximation has conformational energies on the $t=0$ surface as shown in column 3 . The spacing between and ordering of conformational energies is similar to the original MM2 surface. Columns $4-6$ show the change in conformational energies as a function of smoothing. Increase in smoothing is characterized by a reduction in the conformational energy spacing between minima and a rearrangement of the rank ordering of minima, i.e., for $0.001<t<$ 0.01 minimum 2 is the lowest in energy.

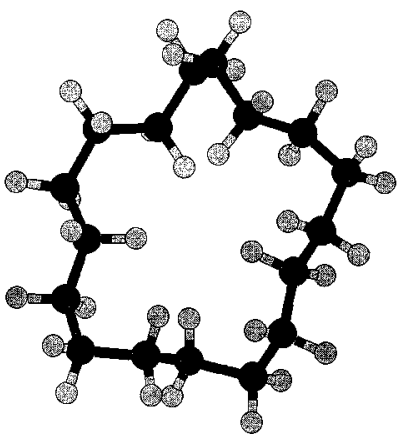

(a)

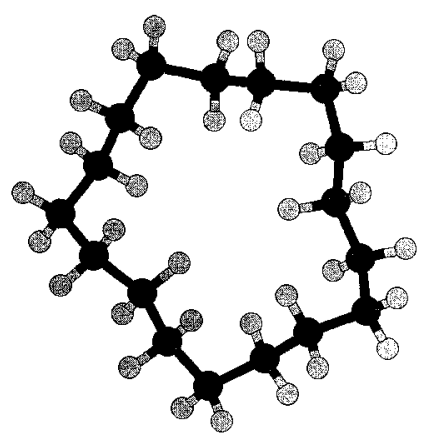

(b)
Figure 4. (a) Global minimum structure for cycloheptadecane with MM2 energy of $19.0680 \mathrm{kcal} / \mathrm{mol}$. (b) Second lowest energy minimum and the structure found by PS-NMLS algorithm with MM2 energy of $19.0774 \mathrm{kcal} / \mathrm{mol}$

which is the structure found using PS-NMLS. This result is to be contrasted with the deterministic nature of the PS-NMLS protocol that converges to the second lowest minimum without regard to the starting structure.

5. Optimizations in $\boldsymbol{\Gamma}_{\mathrm{vr}}$. We applied PS-NMLS in $\Gamma_{\mathrm{vr}}$ for docking two rigid canonical $\alpha$-helices of $\mathrm{CH}_{3} \mathrm{CO}-(\mathrm{L}-\mathrm{Ala})_{9}-$ $\mathrm{NHCH}_{3}$. The variables are the six rigid body degrees of freedom for each helix. Since formation of complexes involves rigid molecules, we optimize only the intermolecular interactions in $\Gamma_{\mathrm{vr}}$. Considerable work has been done on energetic approaches to determine the packing of polyalanine $\alpha$-helices. ${ }^{62}$ It was shown that lower energy packing orientations are minor variations of antiparallel arrangements. These studies also show that the most important contributions to packing of polyalanine helices are from van der Waals interactions with minor contributions from electrostatic interactions. Helix packing has also been studied based on the packing preferences of side chains attached to model helical backbones. ${ }^{64-66}$ These analyses are 


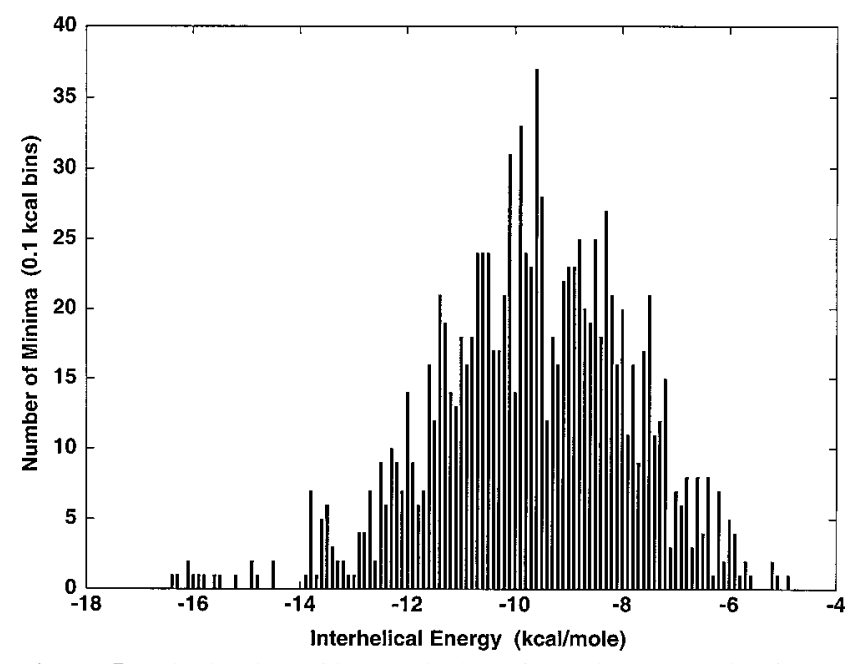

Figure 5. Distribution of interhelical conformational energies for the 1093 unique minima found from an extensive grid search over 18000 unique starting positions for two rigid capped $\mathrm{CH}_{3} \mathrm{CO}-(\mathrm{L}-\mathrm{Ala})_{10^{-}}$ $\mathrm{NHCH}_{3} \alpha$-helices. The global minimum has an energy of -16.4124 $\mathrm{kcal} / \mathrm{mol}$.

based on either a knobs-in-holes ${ }^{63,65}$ or ridges-in-grooves ${ }^{64}$ picture of helix packing.

The orientation of two packed helices with respect to each other can be computed in terms of two parameters, the distance of closest approach $d$ and the packing angle $\Omega$. The distance of closest approach is the shortest distance between two points on the two helix axes. $\Omega$ is the angle between the two helix axes when projected onto a plane normal to the line of closest contact. It is computed as the dihedral angle defined by the points $N_{\mathrm{t} 1}, c p_{1}, N_{\mathrm{t} 2}$, and $c p_{2}$, where $N_{\mathrm{t} i}$ is the N-terminus of helix $i$ and $c p_{i}$ is the point on helix $i$ which is along the line of contact. $\Omega$ varies from $-180^{\circ}$ to $180^{\circ}$; i.e., the helices are exactly parallel if $\Omega=0$ and antiparallel if $\Omega= \pm 180^{\circ}$.

For rigid body optimizations the translational coordinates are defined in terms of the center of mass and the rotations are the Euler angles that describe independent rotations. We use an "xyz-convention" 66 to define the Euler angles. All calculations were done in vacuo and electrostatic terms were excluded.

$\Gamma_{\mathrm{vr}}$ was extensively searched to locate minimum energy conformations for the two polyalanine helices. A grid of 18000 starting conformations was generated by varying the distance between the centers of mass, the packing angle between $-180^{\circ}$ and $180^{\circ}$ in $20^{\circ}$ increments, and the rotation angles about each helix axis from $0^{\circ}$ to $360^{\circ}$ in $40^{\circ}$ increments. The network of 18000 structures includes an even sampling about the parallel, antiparallel, and perpendicular orientations. The conformational energy for each of these starting structures was minimized and duplicate minima removed to yield a set of 1093 unique minima. We used a quasi-Newton method for all minimizations with an rms gradient convergence criterion of $0.0001 \mathrm{kcal} / \mathrm{mol} /$ degree of freedom. The distribution of energies for the 1093 conformations is shown in Figure 5. The global minimum from this grid search has an interhelical van der Waals energy of $V=$ $-16.4124 \mathrm{kcal} / \mathrm{mol}, d=7.16 \AA$, and $\Omega=153.33^{\circ}$. These values correspond to an antiparallel arrangement of the helices with an approximately $30^{\circ}$ twist and denotes a "class a" type of packing of helices described by Walther et al. ${ }^{65}$ which is typical for helices from globular proteins. Table 9 show values of $d, \Omega$, and $V_{12}$ for the 15 lowest minima found from the grid search.

PS-NMLS was tested to see if it could find the global minimum energy conformation for docking the two polyalanine
TABLE 9: Fifteen Lowest Energy Conformers for Docked Polyalanine Helices in Descending Order of Interhelical Energies $^{a}$

\begin{tabular}{ccrc}
\hline minimum & $\begin{array}{c}\text { interhelical energy } \\
(\mathrm{kcal} / \mathrm{mol})\end{array}$ & $\Omega(\mathrm{deg})$ & $d(\AA)$ \\
\hline 1 & -16.4124 & 153.33 & 7.1624 \\
2 & -16.2582 & 149.82 & 6.9569 \\
3 & -16.0996 & 159.79 & 7.1126 \\
4 & -16.0570 & 146.58 & 6.9445 \\
5 & -16.0258 & -32.93 & 6.9680 \\
6 & -15.8974 & -28.92 & 7.1235 \\
7 & -15.7974 & -25.24 & 7.0791 \\
8 & -15.5851 & 155.42 & 7.2866 \\
9 & -15.2452 & 157.45 & 7.3249 \\
10 & -15.5117 & 153.96 & 7.1943 \\
11 & -14.9027 & 161.98 & 7.0153 \\
12 & -14.8666 & 151.80 & 7.4602 \\
13 & -14.7943 & 158.09 & 7.1202 \\
14 & -14.5419 & 150.36 & 7.1636 \\
15 & -14.4517 & -26.56 & 6.7842
\end{tabular}

${ }^{a}$ The table shows the values for the interhelical energies, the packing angles $\Omega$, and the distance of closest approach $d$.

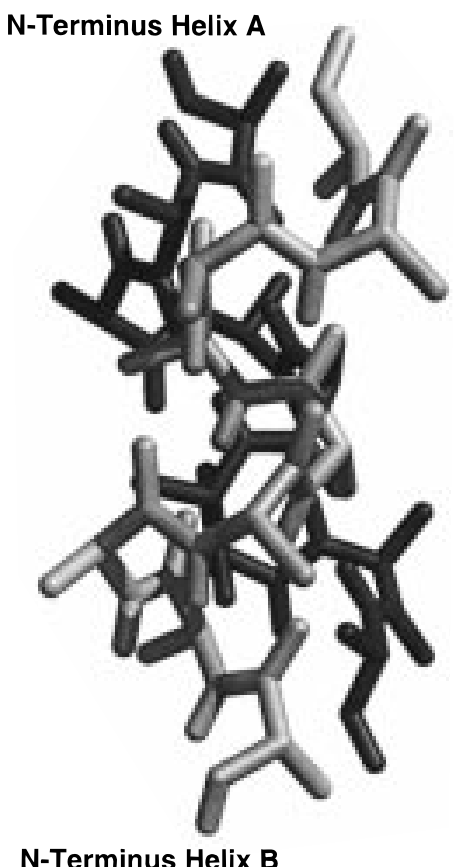

Figure 6. Conformation of the global energy minimum for the packing of two capped, right-handed $\alpha$-helices of sequence $\mathrm{CH}_{3} \mathrm{CO}-(\mathrm{L}-\mathrm{Ala})_{10}-$ $\mathrm{NHCH}_{3}$.

helices. We used a maximum deformation of $t_{\mathrm{d}}=4.0$ beyond which the calculation is numerically unstable without use of a constraining basin function. We set $n_{\mathrm{d}}=150$ and $s=3$ for the smoothing schedule. Local searches are performed for all values of $t<t_{\mathrm{d}}$ during the reversing schedule along the 12 eigenvectors corresponding to the 12 degrees of freedom in $\Gamma_{\mathrm{vr}}$. The global minimum on the $t=4.0$ surface has orientation parameters of $D=20.90 \AA$ and $\Omega=125.86^{\circ}$. Upon returning to the undeformed surface, the PS-NMLS method finds the global minimum obtained from the grid search. This result is obtained reproducibly from any arbitrary starting structure. The global minimum conformation of the docked polyalanine helices is shown in Figure 6.

\section{Discussion}

Features of Potential Smoothing. We illustrate some typical features of the potential smoothing paradigm using onedimensional slices of a rugged PES shown in Figures 7 and 8. 


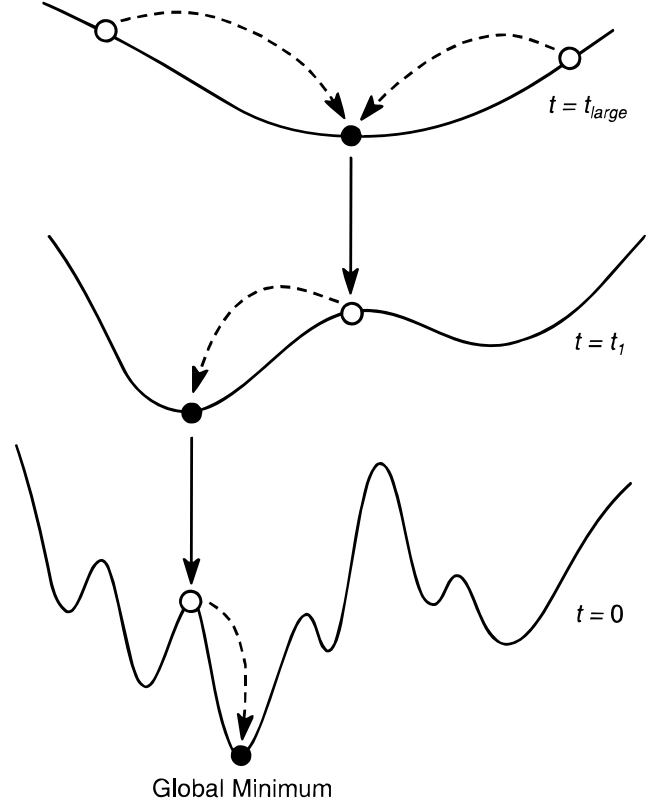

Figure 7. One-dimensional schematic of the effect of a smoothing protocol on a potential energy surface. The original PES is transformed by successive application of a smoothing operator, where the extent of smoothing is dictated by a control parameter $t$. The unsmoothed original surface $(t=0)$, the surface at an intermediate level of smoothing $(t=$ $\left.t_{1}\right)$ and a highly smoothed surface $\left(t=t_{\text {large }}\right)$ are shown. As the surface is transformed, higher lying minima merge into catchment regions of low-lying minima and barriers between minima are progressively lowered. Open circles are starting or intermediate points on each surface. Solid circles are local minima. Dashed arrows show the result of local optimization ending at a local minimum. Solid arrows represent adiabatic movement from a local minimum on one surface to the corresponding starting point on a rougher surface. A simple smoothing protocol consists of repeated cycles of local optimization followed by adiabatic transfer to the next surface.

The process of transforming a potential surface via smoothing is characterized by three kinds of events: mergers, crossings, and shifting of minima.

Two unique minima on the undeformed surface can merge into a common basin at some level of smoothing. The multiple minimum problem is circumvented by reducing the number of minima through a series of such mergers with increasing $t$. Consider a smoothing level $t=t_{1}$ where two formerly distinct minima $M_{i}$ and $M_{j}$ merge into the same basin identified by a common structure and conformational energy. If at $t=t_{1}=$ $\Delta t$ during a smoothing reversal protocol the two minima reappear and a minimization leads to $M_{i}$, then we label the basin at $t=t_{1}$ as $M_{i}$; i.e., $M_{j}$ merges into $M_{i}$ at $t=t_{1}$. If $M_{i}$ and $M_{j}$ are equal in depth and width on the undeformed surface then a merger of these two minima results from eliminating the barrier and an equal translation of the two minima into the new basin. If the energy gap between the two minima is pronounced, then the higher lying minimum slides into the broader basin of the lower lying minimum; i.e., the position of the new single minimum is close to the location of the lower minimum from the undeformed surface. Figure 9 shows the rate at which the number of minima are reduced for cycloheptadecane as function of increasing deformation. For a smoothing process fully characterized by mergers, as shown in Figure 7, the minimum that is projected out on a highly smoothed surface is related to the global minimum and a reversal protocol will find the global minimum.

A second feature of potential smoothing is the crossing of relative energies of two minima. Two unique minima A and B

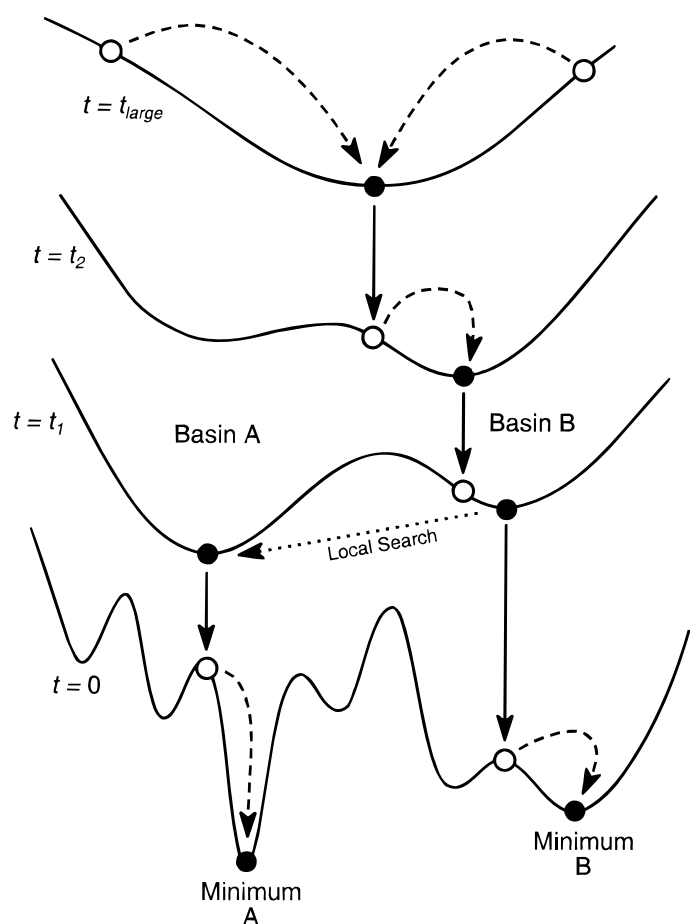

Figure 8. Schematic of a more realistic potential smoothing protocol for molecular search problems. This figure shows a crossing between the two surviving minima on the $t=t_{2}$ surface. A reversing schedule encounters the first bifurcation at $t=t_{2}$. At this level of smoothing the protocol favors basin B over basin A due to a crossing of relative energies which is an artifact of the averaging process. If bifurcations are sampled where the relative energies of the alternative basins are inverted from the $t=0$ surface, then the simple method will not converge to the global minimum. Between $t=t_{2}$ and $t=0$ there exist values of $t$ for which the energy ordering resembles that of the original PES. A local search process coupled to the smoothing schedule can potentially recognize errors due to earlier energy crossings. For example, a local search represented by the dotted arrow on the $t=t_{1}$ surface would correctly decide that basin A should be favored over basin B.

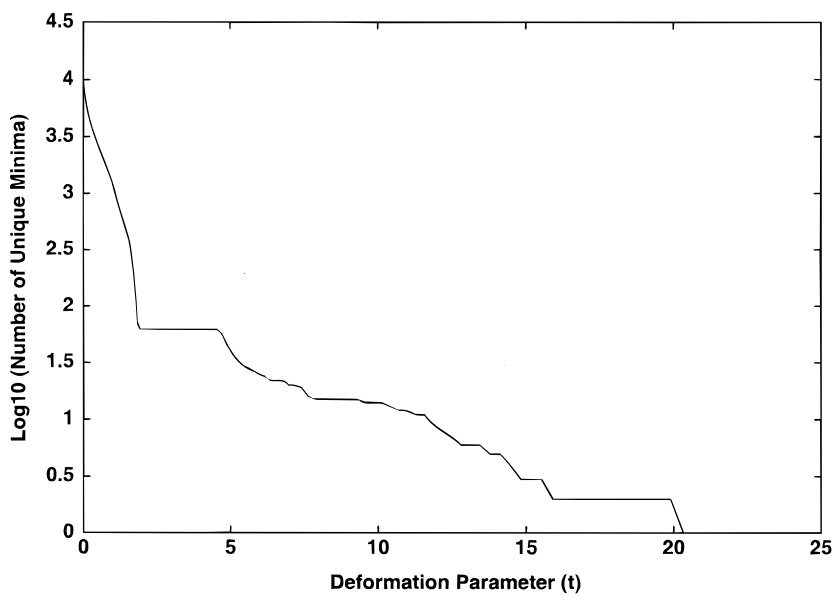

Figure 9. Reduction in the number of minima for cycloheptadecane as a function of increasing PES deformation. On the undeformed surface there are 20469 unique minima. These minima merge into a single minimum on the $t=25$ PES. The figure shows a plot of the $\log _{10}$ of the number of unique minima remaining on the PES as a function of increasing smoothing, $t$.

on the undeformed surface can have conformational energies $V_{\mathrm{A}}$ and $V_{\mathrm{B}}$ such that $V_{\mathrm{A}}<V_{\mathrm{B}}$ for some $t=t_{1}$. For $t_{2}=t_{1}+$ $\Delta t$ these two minima persist as unique conformations but their relative energies can be reversed so that $V_{\mathrm{A}}>V_{\mathrm{B}}$. The effect of crossings on a reversal schedule is shown in Figure 8. As the deformation is slowly reduced, minima that merged at a 
higher level of smoothing reappear at distinct locations and separated by a small barrier; i.e., the reversal protocol sees a bifurcation. In Figure 8 the minimum in basin $B$ is lower in energy than minimum $A$ on the $t=t_{2}$ surface. Because the lower minimum at $t=t_{2}$ is an artifact of a previous energy crossing at some $t_{1}<t<t_{2}$, the DEM reversal procedure will remain trapped in basin $\mathrm{B}$ and not converge to the global minimum. There has been some speculation that crossings are related to the free energy characteristics of the undeformed surface ${ }^{46}$ i.e., the minimum energy basin that becomes energetically favored at large $t$ is related to the free energy minimum on the PES. This proposal is reasonable because narrow deep wells merge into minima defined by broader shallow wells. Generally, the minima that survive on largely deformed surfaces tend to be entropically favored.

A third feature of smoothing is shifting in the location of minima and an initial increase in conformational energies associated with minima as the level of smoothing increases. This is a direct consequence of changing the local curvature of minima and lowering barriers, so minima become more shallow and the basins become broader with an increase in deformation. Figures 7 and 8 show this shifting property as a function of an increase in smoothing.

Figure 8 also shows how a PSS protocol works in correcting certain kinds of energy crossings. At $t=t_{1}$ a reversal protocol will first locate minimum B. Local search starting from this minimum will explore neighboring basins. If basin $\mathrm{A}$ is adjacent to basin B on the smoothed surface, then a normal mode or transition state directed activation followed by minimization can locate the lower energy minimum A. Subsequent return to the undeformed surface will then correctly locate the global minimum in basin A. If the crossing between a pair of minimum energy basins is introduced for a large value of $t$ then a local search protocol will most likely find the lower alternate minimum during the reversal. If there are other crossings on surfaces of very small $t$, a local search may not be able to correct the energy crossing because the barrier heights and surface roughness are already comparable to those of the undeformed PES. In essence, "local" search has a greater range of convergence and can correct crossing errors over larger portions of conformational space on smoother surfaces. This reasoning explains why PS-NMLS in $\Gamma_{\mathrm{vc}}$ finds very low energy minima, but not the absolute global minima, when applied to longer chains of capped polyalanine and cycloheptadecane. For crossings built in at very small values of $t$, a reasonable degree of local search may not be able to sample the global minimum region.

Potential smoothing can be thought of as a projection method; i.e., an important catchment region is projected out by reducing barriers between minima. In direct contrast, barriers are present throughout a simulated annealing protocol and the global minimum is located by generating a trajectory that uses thermal activation to move over barriers. Since the number of barriers to be negotiated by simulated annealing grows exponentially with the size of the system, projection methods are in principle more efficient for larger problems.

Other Variants of Search-Enhanced Potential Smoothing. We have shown in this work that two types of secondary search schemes can be coupled to DEM for improved sampling of conformational space. NMLS or TSBS methods are not the only search methods that can be coupled to potential smoothing for improved searching. Increased sampling on a smooth PES could also be facilitated by a trajectory- or quench-based search mechanism. These include molecular dynamics (MD), ${ }^{67}$ meth-
TABLE 10: Smoothing Parameters $t=t_{\text {detour }}$ at Which Alternate Minima Were Obtained Using a NMLS Protocol in $\boldsymbol{\Gamma}_{\mathrm{vt}}$ for Varying Lengths of Capped Polyalanine $\mathrm{CH}_{3} \mathrm{CO}-(\mathrm{L}-\mathrm{Ala})_{n}-\mathrm{NHCH}_{3}$ Chains

\begin{tabular}{rcc}
\hline$n$ & $t_{\text {detour }}$ & $n_{\mathrm{d}}$ \\
\hline 6 & 0.0223 & 200 \\
7 & 0.0391 & 200 \\
8 & 0.0577 & 300 \\
9 & 0.0792 & 300 \\
10 & 0.0854 & 50 \\
11 & 0.1061 & 350 \\
12 & 0.1179 & 350
\end{tabular}

${ }^{a} t_{\mathrm{d}}=10.0$ and $s=5$ in eq 8 of the text.

ods to promote conformational variation such as poling, ${ }^{68}$ local enhanced sampling (LES), ${ }^{69}$ and Monte Carlo minimization $(\mathrm{MCM})^{10}$ or basin hopping. ${ }^{11}$

Wawak et al. ${ }^{70,71}$ have coupled an MCM method to two types of potential smoothing algorithms, DEM and the distance scaling method of Pillardy and Piela. 57,72 MCM-enhanced potential smoothing algorithms were used successfully for $a b$ initio prediction of crystal packing of hexasulfur and benzene molecules. ${ }^{71}$ Smooth surfaces generated by either DEM or DSM were searched using the basin hopping MCM algorithm. Minima generated using MCM on a smooth surface can be followed back to the undeformed surface using a DEM or DSM reversal protocol.

Computational Efficiency: PS-NMLS versus DEM. In a system with no distance cutoffs on the range of nonbonded interactions, the CPU time for a single energy/gradient calculation scales as $O\left(N^{2}\right)$, where $N$ is the size of system. An efficient local optimization procedure will typically require CPU time that scales as roughly $O\left(N^{3}\right)$. The CPU time for DEM scales approximately as $2 n_{\mathrm{d}}\left[O\left(N^{3}\right)\right]$, where $n_{\mathrm{d}}$ is the number of points along the smoothing schedule. As described above, local search algorithms are essential for correcting errors due to energy crossings between minima. The CPU time in PS-NMLS increases relative to DEM due to the increase in the number of extra $O\left(N^{3}\right)$ minimization calculations. The increase is determined by the number of search directions chosen and the number of points along the reversal schedule for which local searches are performed. In the most extreme case, all possible normal modes, $O(N)$ of them in general, could be searched. The CPU time for this kind of extensive local search PS-NMLS scales as $O\left(N^{4}\right)$. For many problems, we have had success in locating very low or global minimum energy structures using as few as 3-5 search modes, reducing the computational complexity to a small multiple of that for DEM. We are currently developing various time saving strategies for implementing PS-NMLS protocols, one of which is outlined below. Other improvements to PS-NMLS, including coupling local search methods to adiabatic Gaussian density annealing (AGDA) ${ }^{35,55}$ and generating families of low-energy structures instead of a single estimate of the global minimum, are also in progress.

Selecting Smoothing Windows for Local Searches. An obvious method for increasing computational efficiency is based on shortening the smoothing window over which NMLS is performed. Table 10 shows the largest value of deformation, termed $t_{\text {detour, }}$, at which NMLS found alternate lower minima for varying lengths of polyalanine chains in $\Gamma_{\mathrm{vt}}$. Values for $t_{\text {detour }}$ increase as a function of the length of the chain, which is appropriate since the conformational energy surfaces for the larger peptides become increasingly rough and crossings can occur on highly deformed surfaces. Figure 10 shows shows a plot of $t_{\text {detour }}$ as a function of peptide chain length, $n$. The linear 


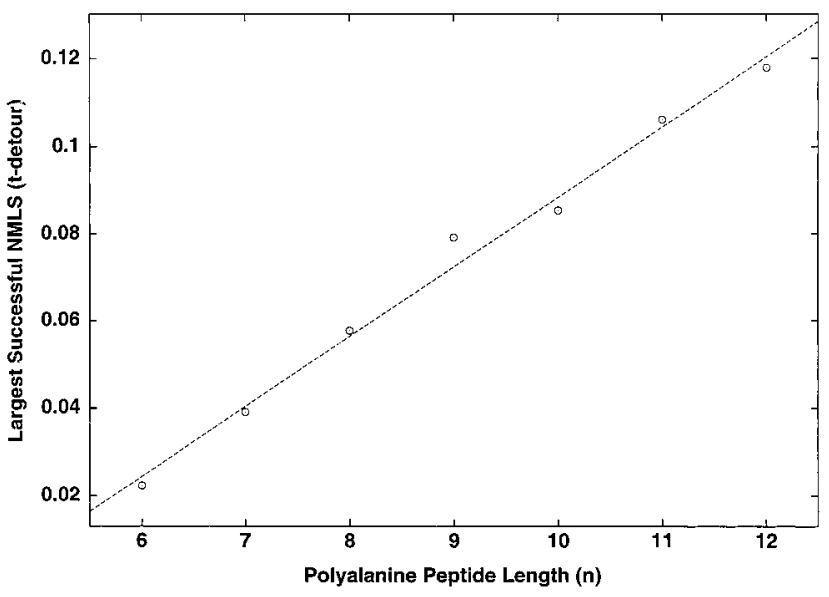

Figure 10. A first-order least-squares fit to the smoothing parameter $t_{\text {detour }}$ as a function of $n$ for application of PS-NMLS to $\mathrm{CH}_{3}-(\mathrm{L}-\mathrm{Ala})_{n}-$ $\mathrm{NHCH}_{3}$ sequences in $\Gamma_{\mathrm{vt}}$ for $(n=5-12)$. The fit could be used to implement windowing schemes to estimate smoothing values for which an NMLS search protocol is to be used in $\Gamma_{\mathrm{vt}}$. Restricting local search to a limited window of $t$ values allows a reduction in computational overhead by eliminating unnecessary and redundant local searches.

least-squares fit to this data gives $t_{\text {detour }}=0.016 n-0.0716$ with a correlation coefficient of 0.995 . An accurate estimate of $t_{\text {detour }}$ provides an upper bound on the window of $t$ values for which NMLS will be efficacious. For example, polyalanine of length $n=8$ has a $t_{\text {detour }}$ value of 0.0577 . Then a PS-NMLS protocol with $n_{\mathrm{d}}=300, s=5$, and $t_{\mathrm{d}}=10.0$ will require about a factor of 3 fewer $O\left[N^{3}\right]$ minimizations using the above $t_{\text {detour }}$ value instead of 5.0 as an upper bound on the range of $t$ values where NMLS will be used. This will translate directly into a factor of 3 savings in overall CPU time required.

Summary. Our results demonstrate that potential smoothing coupled to appropriate modulation and control mechanisms can be a useful tool for global optimization problems. This assertion is substantiated through the successful application of a set of local search enhanced potential smoothing protocols to a cross section of conformational problems that vary in size and complexity of the underlying PES. We have also analyzed limitations of a class of local search methods used to correct errors inherent in smoothing protocols, and demonstrated in simple terms the effect of smoothing on a typical rough PES.

Acknowledgment. This work was supported by a grant from the DOE Environmental Science Management Program. All programs used for calculations reported in this work are part of the TINKER molecular modeling package and are available from the authors. R.V.P. thanks Dr. Gerard T. Barkema for helpful discussions and Prof. Garland R. Marshall for support.

\section{Appendix A}

In this section we list formulas for diffusional smoothing of DOPLS and MM2 potential function terms. The original undeformed functional forms are the initial conditions for diffusion.

1. Bond Length and Bond Angle Terms. For a onedimensional harmonic potential of the form $K_{\mathrm{b}}\left(x-x_{0}\right)^{2}$ the diffusion equation solution is of the form $K_{\mathrm{b}}\left(x-x_{0}\right)^{2}+$ $2 K_{\mathrm{b}} D_{\text {bond }} t$.

2. Torsional Energy Terms. Torsional energies are computed as a sum of one-, two-, and threefold sinusoidal barriers. The functional form is $1 / 2 \sum_{j} V_{j}(1+\cos (j \omega+\phi))$, where $V_{j}$ is the amplitude (one-half the barrier height), $\phi$ is the phase factor which determines the location of the minima, and $\omega$ is the torsional angle. The diffusion equation solution for torsional potentials is of the form $1 / 2 \sum_{j} V_{j}(1+\cos (j \omega+\phi)$ exp$\left.\left(-D_{\text {torsion }} j^{2} t\right)\right)$. This is a solution to a diffusion equation in torsional space in terms of the angular coordinates $\omega$.

In $\Gamma_{\mathrm{vt}}$ the torsional potential can be rewritten in terms of a 1-4 distance, $r_{14}$, using the relation

$$
\cos (\omega)=\frac{2 r_{14}^{2}-(A+B)}{A-B}
$$

where $A$ is the minimum value of the $r_{14}$ distance at $\omega=0$ and $B$ is the maximal distance for $\omega=180$. This form is valid only when the $1-2$ and $1-3$ distances remain fixed. Substitution into the original torsional potential, ${ }_{1} /{ }_{2} \sum_{j} V_{j}(1+\cos (j \omega+$ $\phi)$ ), gives an expression for the energy as a higher order polynomial in $r_{14}$. The smoothing of polynomial functions results in smoothed functions that are polynomials of the same degree as the undeformed function, as illustrated for the covalent terms discussed above. Similarly, the smoothed forms for trigonometric functions are trigonometric functions scaled by a function of the smoothing parameter. Therefore, the qualitative nature of a smoothed 1-4 distance potential for the torsions is similar to smoothed form in terms of the torsions, provided the $1-4$ distance potential is a good approximation to the Fourier series torsional potential.

In $\Gamma_{\mathrm{vc}}$, however, the 1-4 distance potential becomes very complicated because the change in $1-4$ distance is coupled to changes in the $1-2$ and $1-3$ distances. It is not possible to obtain a closed form for the undeformed potential in terms of the 1-4 distance. We therefore smooth the torsional potential in torsional space and scale the smoothed potential relative to its distance space counterparts, i.e., the nonbonded electrostatic and van der Waals terms, through a choice of diffusion coefficients.

3. van der Waals Potential. A two-Gaussian approximation to a 12-6 Lennard-Jones or exp-6 Buckingham function is written as a sum of two Gaussians centered about the origin. The form of these Gaussians is $F_{\mathrm{vdw}}\left(r_{i j}, t=0\right)=a_{i j} \exp \left(-b_{i j} r_{i j}{ }^{2}\right)$. The three-dimensional distance space diffusion equation in $r_{i j}$ $\in(0, \infty)$ is of the form

$$
\frac{\partial F_{\mathrm{vdw}}\left(r_{i j}, t\right)}{\partial t}=D_{\mathrm{vdw}}\left\{\frac{\partial^{2} F_{\mathrm{vdw}}\left(r_{i j}, t\right)}{\partial r_{i j}^{2}}+\frac{2}{r_{i j}} \frac{\partial F_{\mathrm{vdw}}\left(r_{i j}, t\right)}{\partial r_{i j}}\right\}
$$

The solution is of the form

$$
F_{\mathrm{vdw}}\left(r_{i j}, t\right)=\sum_{k=1}^{n_{\text {gauss }}} \frac{a_{i j}}{\left(1+4 D_{\mathrm{vdw}} t\right)^{3 / 2}} \exp \left(-\frac{b_{i j} r_{i j}^{2}}{1+4 D_{\mathrm{vdw}} t}\right)
$$

4. Coulomb Potential. For a Coulomb potential $V\left(r_{i j}\right)=$ $1 / r_{i j}$ as "initial condition" in three dimensions, the diffusion equation solution is of the form

$$
F_{\text {charge }}\left(r_{i j}, t\right)=\frac{1}{r_{i j}} \operatorname{erf}\left(\frac{r_{i j}}{2 D_{\text {charge }} t^{1 / 2}}\right)
$$

This formula, adapted from the work of Amara and Straub ${ }^{34}$ and Moré and $\mathrm{Wu},{ }^{73}$ satisfies the three-dimensional distance space diffusion equation of the form

$$
\frac{\partial F_{\text {charge }}\left(r_{i j}, t\right)}{\partial t}=D_{\text {charge }}\left\{\frac{\partial^{2} F_{\text {charge }}\left(r_{i j}, t\right)}{\partial r_{i j}^{2}}+\frac{2}{r_{i j}} \frac{\partial F_{\text {charge }}\left(r_{i j}, t\right)}{\partial r_{i j}}\right\}
$$




\section{Appendix B}

Many of the standard molecular mechanics potential energy functions such as CHARMM, AMBER, or OPLS can be written in the general form

$$
V_{\text {total }}=V_{\text {bond }}+V_{\text {angle }}+V_{\text {torsion }}+V_{\text {improper }}+V_{\text {vdw }}+V_{\text {charge }}
$$

In potential smoothing, each of these terms is transformed according to a diffusion equation of the form

$$
\frac{\partial F_{\alpha}}{\partial t}=\frac{\partial^{2} F_{\alpha}}{\partial x^{2}}
$$

which is the one-dimensional analog of the multidimensional diffusion operator. Here $F_{\alpha}(x, 0)=V_{\alpha}(x)$ and $\alpha$ refers to one of the six terms in eq B1. Using an equation of the form (B2) with identical diffusion coefficients $D$ for each of the energy functions is equivalent to claiming that each of these functions has similar distance ranges and energy scales. Values for effective diffusion coefficients can be chosen to scale the relative smoothing of each of the energy functions.

The bond term and the nonbonded van der Waals and Coulomb interactions are written in terms of Cartesian distances between the atoms that are part of these interactions. The angle term and torsional terms can be written in terms of the corresponding angular coordinates. From the standpoint of a classical diffusion equation, each of the terms represents different initial conditions for diffusion. The range of the potentials determines the extent of diffusion space within which the solutions of eq B2 need to be obtained. One way to impose different diffusion spaces would be to set appropriate inner and outer boundary conditions for the different initial conditions in eq B1. An alternative method is to compute effective diffusion coefficients for each of the terms in (B1) that are determined by the range of the interaction potential. The effective coefficients may be scaled relative to the values for the van der Waals and Coulomb terms which are set to unity since these nonbonded interactions are of longer range than the local geometry terms.

Effective diffusion coefficients can be computed by a simple comparison of the extents of diffusion spaces. We set $D=1$ for the nonbonded terms which have the largest diffusion extents in diffusion space. In descending order the extent in distance space for the torsions, angles, and bonds follow the order of $r_{\mathrm{nb}}$ $>r_{\text {torsion }}>r_{\text {angle }}>r_{\text {bond. }}$. Accordingly, the diffusion of the geometry terms scaled relative to the nonbonded terms follows the relation

$$
\frac{D_{\mathrm{geom}}}{D_{\mathrm{nb}}}=\frac{r_{\mathrm{geom}}^{2}}{r_{\mathrm{nb}}^{2}}
$$

We set $D_{\mathrm{nb}}=1$ and choose $r_{\mathrm{nb}}$ and $r_{\text {geom }}$ to correspond to typical upper limits in the DOPLS force field for the nonbonded distance and individual geometry distances. One set of choices for these values leads to the diffusion coefficients shown in Table 1. It should be stressed that this is purely an empirical choice, but a necessary one because it draws an exact analogy with the mechanism of diffusion and provides a simple method for modulating the smoothing of diverse potential function terms within a molecular mechanics formulation.

\section{References and Notes}

(1) Leach, A. R. Rev. Comput. Chem. 1991, 2, 1.

(2) Scheraga, H. A. Rev. Comput. Chem. 1992, 3, 73.
(3) Horst, R.; Tuy, H. J. Opt. Theor. Appl. 1987, 54, 253.

(4) Némethy, G.; Scheraga, H. A. Biopolymers 1965, 3, 155.

(5) Griewank, A. O. J. Opt. Theor. Appl. 1981, 34, 11.

(6) Törn, A.; Zilinskas, A.; Zilinskas, A. Global Optimization; Lecture Notes in Computer Science, Vol. 350; Springer-Verlag, Berlin, 1989; pp $117-151$.

(7) Kirkpatrick, S.; Gelatt, C. D.; Vecchi, M. P. Science 1983, 220, 671.

(8) Metropolis, N.; Rosenbluth, A. W.; Rosenbluth, M. N.; Teller, A. H.; Teller, E. J. J. Chem. Phys. 1953, 21, 1087

(9) Berg, B. A.; Neuhaus, T. Phys. Lett. B 1991, 267, 249. 6611.

(11) Wales, D. J.; Doye, J. P. K. J. Phys. Chem. A 1997, 101, 5111.

(12) Li, Z.; Laidig, K. E.; Daggett, V. J. Comput. Chem. 1998, 19, 60.

(13) Guarnieri, F.; Still, W. C. J. Comput. Chem. 1994, 15, 1302.

(14) Saunders, M. J. Comput. Chem. 1989, 10, 203.

(15) Brünger, A. T.; Kuriyan, J.; Karplus, M. Science 1987, 235, 458.

(16) van Laarhoven, P. J. M.; Aarts, E. H. L. Simulated Annealing. Theory and Applications; Kluwer Academic Publishers: Amsterdam, 1987.

(17) Straub, J. E. In Recent Developments in Theoretical Studies of Proteins; Elber, R. E., Ed.; World Scientific: Singapore, 1996; p 137.

(18) Wilson, S. R.; Cui, W. Biopolymers 1990, 29, 225.

(19) Brünger, A. T.; Rice, L. M. Methods Enzymol. 1997, 277, 243.

(20) Ingber, L. Math. Comput. Model 1989, 12, 967.

(21) Wang, Z.; Pachter, R. J. Comput. Chem. 1997, 18, 323

(22) Tsallis, C. J. Stat. Phys. 1988, 52, 479.

(23) Tsallis, C.; Stariolo, D. A. Physica A 1996, 233, 395.

(24) Andricioaei, I.; Straub, J. E. Phys. Rev. E 1996, 53, R3055.

(25) Simon, I.; Némethy, G.; Scheraga, H. A. Macromolecules 1978, 11,797 .

(26) Pincus, M. R.; Klausner, R. D.; Scheraga, H. A. Proc. Natl. Acad. Sci. U.S.A. 1982, 79, 5107.

(27) Dammkoehler, R. A.; Karasek, S. F.; Shands, E. F. B.; Marshall, G. R. J. Comput.-Aid. Mol. Des. 1989, 3, 3 .

(28) Merovitch, H.; Vasquez, M.; Scheraga, H. A. J. Chem. Phys. 1990, 92,1248 .

(29) Goldberg, D. E. Genetic Algorithms in Search, Optimization, and Machine Learning; Addison-Wesley: Reading, MA, 1989.

(30) Hoare, M. R.; McInnes, J. A. Adv. Phys. 1983, 32, 791.

(31) Stillinger, F. H.; Weber, T. J. Stat. Phys. 1988, 52, 1429.

(32) Piela, L.; Kostrowicki, J.; Scheraga, H. A. J. Phys. Chem. 1989 93,3339 .

(33) Ma, J.; Straub, J. E. J. Chem. Phys. 1993, 101, 533.

(34) Amara, P.; Straub, J. E. Phys. Rev., B 1996, 53, 13857.

(35) Ma, J.; Hsu, D.; Straub, J. E. J. Chem. Phys. 1993, 99, 4024

(36) Shalloway, D. In Recent Advances in Global Optimization; Floudas,

C. A., Pardalos, P. M., Eds.; Princeton University Press: Princeton, NJ, 1992; pp 433.

(37) Church, B.; Oresic, M.; Shalloway, D. In Global Minimization of Nonconvex Energy Functions: Molecular Conformation and Protein Folding; DIMACS, Vol. 23; Pardalos, P. M., Shalloway, D., Xue, G., Eds.; Am. Math. Soc.: Providence, RI, 1996; p 41.

(38) Nakamura, S.; Hirose, H.; Ikeguchi, M.; Doi, J. J. Phys. Chem. 1995, 99, 8374 .

(39) Ponder, J. W. TINKER: Software Tools for Molecular Design, Version 3.6; Washington University School of Medicine, 1998.

(40) Weiner, S. J.; Kollman, P. A.; Case, D. A.; Singh, U. C.; Ghio, C.; Alagona, G.; Profeta, S., Jr.; Weiner, P. J. Am. Chem. Soc. 1984, 106, 765.

(41) Jorgensen, W. L.; Tirado-Rives, J. J. Am. Chem. Soc. 1988, 110, 1657

(42) Allinger, N. L. J. Am. Chem. Soc. 1977, 99, 8127.

(43) Brooks, B. R.; Bruccoleri, R. E.; Olafson, B. D.; States, D. J.; Swaminathan, S.; Karplus, M. J. Comput. Chem. 1983, 4, 187.

(44) Moré, J.; Wu, Z. In Global Minimization of Nonconvex Energy Functions: Molecular Conformation and Protein Folding; DIMACS, Vol. 23; Pardalos, P. M., Shalloway, D., Xue, G., Eds.; Am. Math. Soc.: Providence, RI, 1996; p 151.

(45) Kostrowicki, J.; Piela, L.; Cherayil, B. J.; Scheraga, H. A. J. Phys. Chem. 1991, 95, 4113

(46) Kostrowicki, J.; Scheraga, H. A. J. Phys. Chem. 1992, 96, 7442

(47) Amara, P.; Straub, J. E. J. Phys. Chem. 1995, 99, 14840.

(48) Davidon, W. C. Math. Programming 1975, 9, 1.

(49) Ponder, J. W.; Richards, F. M. J. Comput. Chem. 1987, 8, 1016.

(50) Czerminski, R.; Elber, R. J. Chem. Phys. 1990, 92, 5580.

(51) Ulitsky, A.; Shalloway, D. J. Chem. Phys. 1997, 106, 10099

(52) Cerjan, C. J.; Miller, W. H. J. Chem. Phys. 1981, 75, 2800.

(53) Barkema, G. T.; Mousseau, N. Phys. Rev. Lett. 1996, 77, 4358.

(54) Northby, J. A. J. Chem. Phys. 1987, 6, 599.

(55) Tsoo, C.; Brooks III, C. L. J. Chem. Phys. 1994, 101, 6405.

(56) Doye, J. P. K.; Wales, D. J. Phys. Rev. Lett. 1998, 80, 1357

(57) Pillardy, J.; Piela, L. J. Phys. Chem. 1995, 99, 11805. 
(58) Zimmerman, S. S.; Pottle, M. S.; Némethy, G.; Scheraga, H. A. Macromolecules 1977, 10, 1.

(59) Hart, R. K.; Pappu, R. V.; Ponder, J. W., to be submitted for publication.

(60) Saunders, M.; Houk, K. N.; Wu, Y.D.; Still, W. C.; Lipton, M.; Chang, G.; Guida, W. C. J. Am. Chem. Soc. 1990, 112, 1419.

(61) Ngo, J. T.; Karplus, M. J. Am. Chem. Soc. 1997, 119, 5657.

(62) Chou, K. C.; Némethy, G.; Scheraga, H. A. J. Am. Chem. Soc. 1984, 106, 3161.

(63) Crick, F. Acta Crystallogr. 1953, 6, 689

(64) Chothia, C.; Levitt, M.; Richardson, D. J. Mol. Biol. 1981, 145, 215.

(65) Walther, D.; Eisenhaber, F.; Argos, P. J. Mol. Biol. 1996, 255, 536 (66) Goldstein, H. Classical Mechanics; Addison-Wesley: Reading, MA, 1980; Chapter 4 and Appendix B.
(67) Huber, T.; Torda, A. E.; van Gunsteren, W. F. J. Phys. Chem. A 1997, 101, 5926

(68) Smellie, A.; Teig, S. L.; Towbin, P. J. Comput. Chem. 1995, 16 , 17.

(69) Roitberg, A.; Elber, R. J. Chem. Phys. 1991, 95, 9277.

(70) Wawak, R. J.; Gibson, K. D.; Liwo, A.; Scheraga, H. A. Proc. Natl. Acad. Sci., U.S.A. 1996, 93, 1743.

(71) Wawak, R. J.; Pillardy, J.; Liwo, A.; Gibson, K. D.; Scheraga, H. A. J. Phys. Chem. A 1998, 102, 2904.

(72) Pillardy, J.; Piela, L. J. Comput. Chem. 1997, 18, 2040.

(73) Moré, J.; Wu, Z. In Large Scale Optimization with Applications: Molecular Structure and Optimization, Biegler, L. T., Coleman, T. F., Conn, A. R., Santosa, F. N., Eds.; Springer- Verlag: New York, 1997; p 99. 